Check for updates

Cite this: Phys. Chem. Chem. Phys., 2020, 22, 10500

Received 22nd October 2019, Accepted 6th January 2020

DOI: $10.1039 / c 9 c p 05753 f$

rsc.li/pccp

\section{Benchmark and performance of long-range corrected time-dependent density functional tight binding (LC-TD-DFTB) on rhodopsins and light-harvesting complexes $\dagger$}

\author{
Beatrix M. Bold, (D) ${ }^{a}$ Monja Sokolov, ${ }^{a}$ Sayan Maity, (D) ${ }^{\mathrm{b}}$ Marius Wanko, ${ }^{\mathrm{c}}$ \\ Philipp M. Dohmen, ${ }^{a}$ Julian J. Kranz, ${ }^{\text {ad }}$ Ulrich Kleinekathöfer, (D) ${ }^{\text {b }}$ \\ Sebastian Höfener (iD ${ }^{a}$ and Marcus Elstner (iD *ad
}

\begin{abstract}
The chromophores of rhodopsins (Rh) and light-harvesting (LH) complexes still represent a major challenge for a quantum chemical description due to their size and complex electronic structure. Since gradient corrected and hybrid density functional approaches have been shown to fail for these systems, only range-separated functionals seem to be a promising alternative to the more time consuming postHartree-Fock approaches. For extended sampling of optical properties, however, even more approximate approaches are required. Recently, a long-range corrected (LC) functional has been implemented into the efficient density functional tight binding (DFTB) method, allowing to sample the excited states properties of chromophores embedded into proteins using quantum mechanical/molecular mechanical (QM/MM) with the time-dependent (TD) DFTB approach. In the present study, we assess the accuracy of LC-TD-DFT and LC-TD-DFTB for rhodopsins (bacteriorhodopsin (bR) and pharaonis phoborhodopsin (ppR)) and LH complexes (light-harvesting complex II (LH2) and Fenna-Matthews-Olson (FMO) complex). This benchmark study shows the improved description of the color tuning parameters compared to standard DFT functionals. In general, LC-TD-DFTB can exhibit a similar performance as the corresponding LC functionals, allowing a reliable description of excited states properties at significantly reduced cost. The two chromophores investigated here pose complementary challenges: while huge sensitivity to external field perturbation (color tuning) and charge transfer excitations are characteristic for the retinal chromophore, the multi-chromophoric character of the LH complexes emphasizes a correct description of inter-chromophore couplings, giving less importance to color tuning. None of the investigated functionals masters both systems simultaneously with satisfactory accuracy. LC-TD-DFTB, at the current stage, although showing a systematic improvement compared to TD-DFTB cannot be recommended for studying color tuning in retinal proteins, similar to some of the LC-DFT functionals, because the response to external fields is still too weak. For sampling of LH-spectra, however, LC-TD-DFTB is a viable tool, allowing to efficiently sample absorption energies, as shown for three different LH complexes. As the calculations indicate, geometry optimization may overestimate the importance of local minima, which may be averaged over when using trajectories. Fast quantum chemical approaches therefore may allow for a direct sampling of spectra in the near future.
\end{abstract}

\section{Introduction}

Many biological processes are triggered by the absorption of photons. Two prominent and well studied protein classes involving these processes are rhodopsins and light-harvesting (LH) complexes. Microbial rhodopsins consist of seven transmembrane helices (Fig. 2) embedded in a membrane, forming an internal binding pocket, where the chromophore all-trans retinal (Fig. 1) is bonded via a Schiff base (SB) to an $\varepsilon$-amino group of a lysine side chain. They are mainly classified through 
<smiles>CC1=C(/C=C/C(C)=C/C=C/C(C)=C/C=[NH+])C(C)(C)CCC1</smiles>

b)<smiles>[1H][NH+]=C/C=C(C)/C=C\C=C\C=C</smiles>

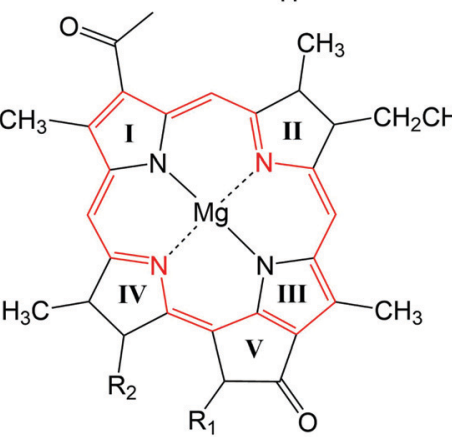

Fig. 1 Top: (a) all-trans retinal, (b) 6-s-cis-11-cis retinal, in blue: twisted dihedral angle (C4-C5-C6-C7), (c) PSB5 model; bottom: BChla; in red diaza ${ }^{18}$-annulene substructure visible; full model: $R_{1}=$ COOMe, $R_{2}=$ phytyl-tail; model with truncated phytyl-tail: $\mathrm{R}_{1}=\mathrm{COOMe}, \mathrm{R}_{2}=\mathrm{COOMe}$ truncated model: $\mathrm{R}_{1}=\mathrm{H}, \mathrm{R}_{2}=\mathrm{CH}_{3}$

their retinal conformation and binding pocket. In organic solvents the absorption maximum of the chromophore retinal is about $450 \mathrm{~nm},{ }^{1}$ while in proteins it varies from 360 to $635 \mathrm{~nm} .{ }^{2}$ As rhodopsin models in this work, bacteriorhodopsin (bR) and pharaonis phoborhodopsin (ppR), are chosen. The binding pockets of $\mathrm{bR}$ and $\mathrm{ppR}$ differ in only 10 amino acids within $5 \AA$ of the retinal and features the same arrangement of counter ions. Despite the similarity, the absorption maximum of $\mathrm{ppR}$ $(497 \mathrm{~nm})^{3}$ is blue shifted by about $70 \mathrm{~nm}$ relative to bR $(568 \mathrm{~nm}){ }^{4}$ which has been attributed to a number of small contributions that all add up in a constructive manner. The overall shift between these two proteins hence poses a critical test as the experimental value is less likely achieved due to error cancellation as, e.g., the shift from vacuum to the protein (opsin shift).,

LH complexes are aggregates of proteins and chromophores, i.e., often bacteriochlorophyll (BChl) or chlorophyll (Chl) chromophores, which are mainly responsible for photosynthesis in bacteria and plants. They absorb light and collect the energy to transfer it to the photosynthetic reaction center (RC) using an excitation energy transfer (EET) mechanism. ${ }^{7}$ The absorption spectra of (bacterio)chlorophylls exhibit two key features, which are the Soret-band in the near UV region and the Q-bands in the visible region. The labeling of the absorption peaks is based on the four-orbital model for porphyrin derivatives proposed by Gouterman et al ${ }^{8,9}$ While in free-base porphyrins the two highest occupied molecular orbitals (HOMOs) and lowest unoccupied molecular orbitals (LUMOs) are energetically degenerated, in (bacterio)chlorophylls this degeneracy is lifted due to the reduced symmetry leading to a splitting of the Q-bands into the so-called $\mathrm{Q}_{x}$ and $\mathrm{Q}_{y}$ bands. In this work, only the $\mathrm{Q}_{y}$ state, i.e., the lowest excited state, is considered. In organic solvents the absorption maximum of BChl a (Fig. 1) is at around $770 \mathrm{~nm} .{ }^{10}$

In the present work, two LH2 complexes of Rs. molischianum and Rps. acidophila and the FMO complex of C. tepidum are chosen (Fig. 2). The LH2 complex of Rs. molischianum (in brackets are given the values for Rps. acidophila) contains 24 (27) BChls, arranged in two rings, called B800 $(1.55 \mathrm{eV})$ and B850 $(1.46 \mathrm{eV})$ corresponding to their respective absorption maxima. The B800 ring consists of eight (nine) BChl a chromophores with an interchromophore distance of about $20 \AA$, while the $\mathrm{B} 850$ ring consists of 16 (18) BChl a chromophores with a distance of about $9 \AA$ A. The FMO complex used here consists of a monomer including seven BChls, which are separated by about $10-20 \AA$ and arranged asymmetrically in contrast to the LH2 complex. The absorption maxima of the FMO complex are found experimentally in a range of $790 \mathrm{~nm}$ to $825 \mathrm{~nm}(1.50-1.57 \mathrm{eV}) .{ }^{11}$ The reason for the red-shift compared to the one in organic solvents $\left(770 \mathrm{~nm}(1.61 \mathrm{eV})^{10}\right)$ is found to be originating from the protein environment and the excitonic inter-chromophore coupling. In the case of LH2, the absorption shift of the B850 ring mainly originates from the exciton couplings, while the absorption shift of the B800 ring and the ones occurring in the FMO complex are to a large extent caused by the protein environment. ${ }^{12-14}$

The individual chromophores in these biological systems exhibit very specific absorption spectra. In principle, there are several factors determining the absorption maximum, which

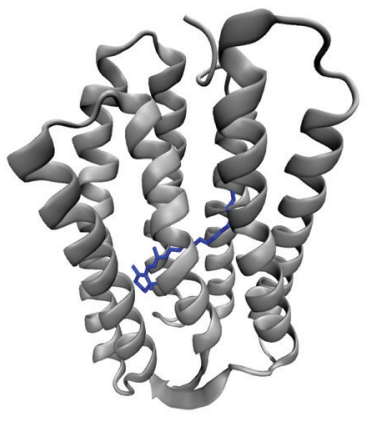

(a)

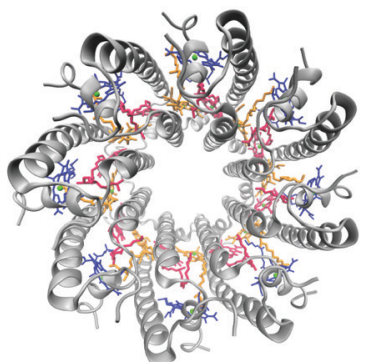

(b)

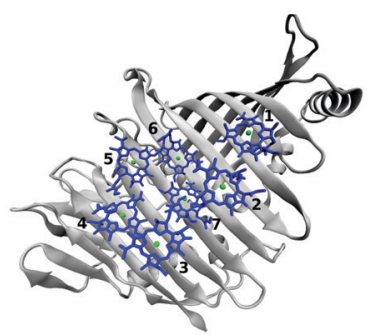

(c)

Fig. 2 (a) Side view of a rhodopsin model, here bR, with retinal in blue. (b) Top view of the $\mathrm{LH} 2$ complex from Rs. molischianum with the BChla chromophores of the B800 ring colored in blue and those belonging to the $B 850$ ring in red and orange. (c) FMO monomer with seven BChla chromophores. 
are (i) the chromophore structure, influenced by the protein environment, (ii) the interaction with the electrostatic environment due to ionic, polar or polarizable groups of amino acids, and (iii) direct hydrogen-bonding or van der Waals (vdW) interactions and (iv) exciton couplings. All these factors are responsible for "color-tuning" of the respective chromophores within their protein environment. The detailed mechanism of "color-tuning" is experimentally not fully available and computational studies are thus of interest for a deeper understanding. Computational methods can disentangle those factors, however the choice of the method is important in order to confirm that the form of the spectrum results from correct physical description and not due to error cancellation. The computational methods should therefore be sufficiently efficient to allow for sampling of the conformational space. Single structures, e.g., computed by $\mathrm{QM} / \mathrm{MM}$ geometry optimization may not be representative for ensemble averages, and information about the peak widths can only be gained from standard deviations.

Both chromophores, retinal and BChl a have been shown to be particularly cumbersome for an accurate quantum chemical description. Often even obtaining ground state geometries is a non-trivial task as various methods yield very different values for the bond-length alternation (BLA), which is defined as the difference between the average single- and double bond lengths along a conjugation chain or ring, and has a sizable impact on excitation energies. Excited states can exhibit multi-reference character, while charge transfer is important, in particular for retinal. In principle, this would require multi-reference configuration interaction methods (MRCI). Complete-active space self-consistent field (CASSCF) together with second-order perturbation theory (CASPT2) yields excited state properties in agreement with experimental results for retinal, however, at high computational costs. ${ }^{15-17}$ An alternative method to study retinal is the $a b$ initio spectroscopy oriented configuration interaction (SORCI) method, which yields the same accuracy as CASPT2 but at reduced computational effort. ${ }^{18}$ In contrast to retinal, $\mathrm{BChl}$ has a large active space, which makes it difficult for MRCI methods to investigate its properties in the excited state. MRCI methods used in previous studies are e.g., RASSCF/ RASPT2 ${ }^{19,20}$ or DFT/MRCI. ${ }^{21}$ Similarly, optimized geometries have to be computed at a computationally less expensive level of theory.

Single-reference methods with in many cases sufficient accuracy such as the second-order approximate coupled cluster singles and doubles (CC2) can describe retinal excited states for a mostly planar retinal configuration or without the inclusion of the $\beta$-ionone ring..$^{22-24}$ The optimization of the retinal geometry in the ground and excited state is only accurate if far away from the conical intersection region. ${ }^{23,25}$ Hence, the popular CC2 method is not applicable to all intermediate steps required when studying, e.g., the isomerization of retinal. In the case of BChl, CC2 can successfully describe the excitation energies of truncated BChl structures, ${ }^{26}$ while it shows weaknesses in the application to the complete BChl structure, as it occurs in proteins due to the sensitivity on the multi-configurational character of BChl. ${ }^{21}$
Density functional theory (DFT) methods, although computationally efficient, can only be applied with care: DFT in the generalized gradient approximation (GGA) significantly underestimates the BLA, while hybrid functionals result in improved predictions of the BLA when an increasing amount of exact exchange is included. ${ }^{6,27}$ Excitation energies obtained with the response formalism (TD-DFT) are only reasonable for mostly planar retinal structures. ${ }^{24}$ The response of the excitation energies on the electrostatic interaction, is grossly underestimated with standard TD-DFT functionals, ${ }^{18}$ making these methods effectively useless for color tuning studies. For BChl, however, hybrid functionals seem to perform reasonably well, when taking DFT/MRCI as reference. ${ }^{21}$ Problems with hybrid functionals occur especially when investigating the carotenoids, which are also present in LH complexes. ${ }^{12}$

The failure of TD-DFT methods, when using standard GGA or hybrid functionals, is rooted with their inability to capture charge transfer excitations which play a role in rhodopsins and to some extend also in LH complexes. ${ }^{14,18}$ For charge transfer excitations in organic molecules, an improvement is provided by long-range corrected (LC) functionals, ${ }^{28,29}$ and even for the isomerization of retinal. ${ }^{30}$ A study investigating the excited state relaxation of retinal shows that $L C-B L Y P^{31}$ provides results in accordance with CASPT2. ${ }^{32}$ In a benchmark study on BChl a in FMO, CAM-B3LYP ${ }^{33}$ was reported to yield results in accordance with DFT/MRCI. ${ }^{21}$ Furthermore, CAM-B3LYP can reproduce experimental results for excitation energies of BChl a in different solvents. $^{34}$

Using $a b$ initio and TD-DFT approaches, excitation energies are often computed for single structures, e.g., for QM/MM optimized geometries of chromophore and protein environment. The computation of excitation energies of sampled structures obtained by molecular dynamics (MD) simulations is required not only to determine correct averages and widths of the absorption spectra, but also to investigate, e.g., temperature effects as reported in previous studies. ${ }^{35,36}$ Therefore, methods with low computing costs based on semi-empirical Hamiltonians are frequently applied. In $\mathrm{LH}$ complexes, ZINDO/S-CIS ${ }^{37,38}$ is often used to sample excitation energies. ${ }^{27,39-41}$ In previous studies on retinal proteins, some of us have used OM2/MRCI for sampling of excitation energies, since its performance for relative excitation energies is comparable to SORCI, ${ }^{18,42}$ while absolute excitation energies are blue shifted. The semi-empirical TD-DFTB method faces the same problems as TD-DFT methods using GGA functionals, since TD-DFTB is based on the PBE exchangecorrelation expression. ${ }^{43}$

Recently, a LC functional has been implemented into the density functional tight binding (LC-DFTB) method and has been extended to the time-dependent scheme (LC-TD-DFTB), which is about three orders of magnitude faster than the LC-TD-DFT method. ${ }^{44,45}$ The newly implemented LC-TD-DFTB allows for extensive sampling of absorption spectra and also for direct propagation of excitons, as recently shown for an anthracene crystal. ${ }^{46}$ It is therefore a promising method to study rhodopsins and LH complexes. LC-TD-DFTB has already been successfully benchmarked for a test set of small organic molecules involving 
charge-transfer excitations ${ }^{45}$ and found its application in the computation of gas-phase relaxation dynamics of cycloparaphenylen molecules. ${ }^{47}$ Since TD-DFTB is based on GGA and does not work for retinal proteins, ZINDO/S-CIS likely overestimates electrostatic effects on BChlabsorption spectra, ${ }^{21}$ a thorough test of LC-TD-DFTB seems desirable. Even though previous studies on both retinal and BChl proteins showed that LC functionals lead to an improved description over standard functionals, ${ }^{21,30,32,36,48,49}$ there is still no systematic benchmark regarding the factors of color tuning.

In the present work, we perform a benchmark study on both the isolated chromophores retinal and BChl a as well as on the biological systems, to determine the accuracy of LC-TD-DFT and LC-TD-DFTB for describing color-tuning effects. The article is structured as follows: First, the benchmark study of LC-TDDFT and LC-TD-DFTB on retinal and BChla is presented on computing excitation energies with respect to geometry effects and electrostatic effects. Second, a benchmark of LC-TD-DFTB for the computation of excitonic couplings is performed. Finally, the performance of LC-TD-DFT and LC-TD-DFTB is demonstrated on two rhodopsins, bR and ppR, and two LH complexes, the LH2 and FMO complex.

\section{Computational details}

\subsection{Models and geometry optimization}

The three retinal model systems used in Section 3, displayed in Fig. 1, were taken from ref. 18. The rhodopsin model systems used in Section 4, bR (PDB code 1C3W) and ppR (PDB code 1H68), were taken from ref. 5. Concerning the chromophore BChla, geometry optimizations were carried out using the Turbomole program package ${ }^{50}$ using HF and DFT, employing the functionals BH-LYP, B3LYP and BLYP together with the Karlsruhe basis sets def2-SV(P), def2-SVP, and def2-TZVP. ${ }^{51,52}$ Additionally, geometry optimizations were carried out at the SCC-DFTB level of theory, ${ }^{53}$ denoted DFTB in the following, and at the DFT level of theory using the range-separated CAM-B3LYP functional together with the def2-SVP and def2-TZVP basis sets, as implemented in the ORCA program package. ${ }^{54}$ For these different geometries, the diaza[18]-annulene substructure of $\mathrm{BChla},{ }^{55}$ see Fig. 1 in red, was used to calculate the BLA. To account for protein environment effects on the excitation energies of BChla, the $\mathrm{QM} / \mathrm{MM}$ optimized structures of the FMO complex (PDB code 3EOJ) of $P$. aesturii were taken from ref. 21.

For the benchmark study (Section 3.2.3) of the exciton couplings of the BChl a chromophores, a model system was constructed. The model system contains a BChl a structure (see Fig. 1), where all substituents are replaced by hydrogen atoms except of the carbonyl group of ring V. This structure was firstly optimized using SCC-DFTB. Subsequently, dimers were build differing in the inter-dimer distance.

The LH complexes used in Section 4 were QM/MM optimized, which are the FMO complex from C. tepidum (PDB code 3ENI) and the LH2 complexes from Rs. molischianum (PDB code 1LGH) and Rps. acidophila (PDB code 1NKZ). All QM/MM optimizations were performed using the DFTB3/3OB method for the QM region as implemented in the GROMACS package (version 2017.1). ${ }^{56,57}$ The QM regions of the FMO complex and the LH2 complex contain a single BChl a chromophore, while the respective phythyl tail is not part of the QM region. Neglecting the phythyl tail in the QM region saves computational cost and does not significantly effect the excitation energies, $c f$. ref. 27 and 39. Both systems have been QM/MM minimized using the steepest descent (SD) algorithm (100000 steps with a tolerance of $1000 \mathrm{~kJ} \mathrm{~mol}^{-1} \mathrm{~nm}^{-1}$ ).

\subsection{Classical and QM/MM MD simulations}

For rhodopsins, QM/MM MD simulations of both models were performed, since failures of force-field-only descriptions of the active site of retinal proteins were previously reported. ${ }^{35,58,59}$ Moreover, a well-chosen QM region is important for retinal proteins to be able to investigate effects like charge transfer, protein polarization and dispersion interaction. ${ }^{6}$ In the present study, the QM region includes retinal, the counter ions near the retinal Schiff base (RSBH+) (D85 and D212 for bR, D75 and D201 for $\mathrm{ppR}$ ), the three water molecules near the RSBH+ and a third amino acid close to the active site (R82 for bR and R72 for ppR), see Fig. 8(b). The remaining parts of the system were treated with MM using the CHARMM36 force field. ${ }^{60}$ The QM/MM MD simulations were carried out with the DFTB3/ 3OB method (extended self-consistent-charge Density-Functional Tight-Binding) $)^{61-63}$ for the QM region, implemented in the GROMACS package. ${ }^{57}$ DFTB3/3OB is known to produce a reliable model of hydrogen bonded structures, which are present in rhodopsins, since it is able to describe hydrogen-bonded networks with similar accuracy as full DFT calculations performed with medium sized basis sets. ${ }^{61}$ The QM/MM MD trajectories had a time length of $1 \mathrm{~ns}$. A QM/MM sampling of the LH complexes would require a multichromophoric QM region, which is not easily possible within the current approach. Therefore, classical MD simulations were performed with a time length of 1 ns. Computational details concerning the equilibration of the systems can be found in the ESI, $\dagger$ see Section S1.1. The classical MD simulations were carried out using the GROMACS package (version 2016.3) ${ }^{64}$ and the CHARMM27 force-field. ${ }^{65}$

\subsection{Excitation energies}

Vertical excitation energies were performed at the HF/CIS, ZINDO/S-CIS, ${ }^{37,38}$ TD-DFT (BP86, B3LYP) and LC-TD-DFT (CAMB3LYP, $\omega$ B97X, LC-BLYP) level of theory as implemented in the ORCA program package. ${ }^{54} \mathrm{ZINDO} / \mathrm{S}$ is a short hand notation for ZINDO/S-CIS and LC-TD-DFT is abbreviated as LC-DFT in the following. For the HF/CIS computations, the def2-SVP basis set ${ }^{51}$ was chosen. For the ZINDO/S calculations an active space of $(10,10)$, i.e., including the 10 highest occupied and the 10 lowest unoccupied states, was employed, which yields a sufficient agreement with experimental results for both $\mathrm{BChl}$ a and retinal. ${ }^{41,66}$

The Tamm-Dancoff approximation (TDA) ${ }^{67}$ has been invoked for all TD-DFT calculations together with the resolution of identity (RI) ${ }^{68}$ to speed up the computations. We used the def2-TZVP basis set $^{52}$ and have considered RIJCOSX ${ }^{69}$ for the Coulomb integral and HF exchange terms as well as def2/J as an 
auxiliary basis $\operatorname{set}^{70}$ in the RI approximation. In order to avoid large errors due to possible charge-transfer contributions in vertical excitations, long-range corrected functionals such as CAM-B3LYP, $\omega \mathrm{B} 97 \mathrm{X}$ and LC-BLYP were employed. These functionals are compared in the present work as they differ in the amount of exact exchange. For example, LC-BLYP contains $0 \%$ exact exchange at short range and $100 \%$ at long-range, ${ }^{31} \omega \mathrm{B} 97 \mathrm{X}$ contains $16 \%$ exact exchange at short range and $100 \%$ at longrange $^{71}$ and CAM-B3LYP consists of 19\% exact exchange at short range and $65 \%$ at long-range. ${ }^{33}$ Vertical excitation energies were furthermore performed using the time-dependent generalization of DFTB (TD-DFTB) ${ }^{72}$ without and with long-range corrected functionals (LC-TD-DFTB). ${ }^{44,45,73}$ LC-TD-DFTB is abbreviated as LC-DFTB in the following. In LC-DFTB, the local BNL functional $^{74,75}$ for the short-range part and a conventional non-local Hartree-Fock exchange for $\omega \rightarrow \infty$ for the longrange part was implemented. ${ }^{46}$ The range-separation parameter $\omega$ is set to $\omega=0.3 / a_{0}$ for the computation of the electronic parameters, which was already used in previous work. ${ }^{46}$ DFTB uses a minimal atomic orbital basis set, which is computed from atomic Kohn-Sham equations, and an additional harmonic potential is introduced to confine the basis function.

The DFT/MRCI calculations were performed with a parallel version of the DFT/MRCI code using an interface to the ORCA program package. ${ }^{76}$ For the $\mathrm{BChl}$ a chromophores we use a protocol similar to that recently reported for a Chl a chromophore. ${ }^{77}$ The first SCF step for vacuum and in the QM/MM framework were performed in ORCA 4.0 using the BH-LYP functional together with a def2-SV(P) split valence basis set. ${ }^{51}$ The latest Hamiltonian (R2018) and the "short" parameter sets were employed with a $0.8 E_{\mathrm{h}}$ threshold for the initial and production DFT/MRCI run with 12 roots required for all structures in the present study. The reference space has been considered as eight HOMOs and six LUMOs including single and double excitations.

In order to provide reference values for vertical excitation energies, wavefunction methods are often applied. Since in the present study large systems are investigated, only low-scaling wavefunction methods could be applied to guarantee applicability for the entire set of models used. Therefore, the reference method for small molecules is a second-order approximate coupled-cluster singles and doubles (CC2) method and the algebraic diagrammatic construction scheme of second order, denoted ADC(2). ${ }^{78,79}$ Both methods employ RI. Since with increasing system size even these efficient excited-state methods can become unfeasible, these schemes are employed in the scaled-opposite-spin approximation in combination with the Laplace transformation (LT) to reduce the computational scaling, i.e., LT-SOS-RICC2 and LT-SOS-RIADC(2). ${ }^{80,81}$ In the following, these methods are denoted SOS-CC2 and SOS-ADC(2) since the RI and LT introduce only deviations significantly below the method error. All these wavefunction based methods are performed using the Turbomole program package. ${ }^{50}$ The vertical excitation energies are based on geometries obtained with the def2-SVP basis set. No significant difference were found when performing, as an exemplary comparison, the same calculations using the def2-TZVP basis set.
For the computation of the vertical excitation energies on the chromophores within their protein environment, the QM region were chosen as described above. The electrostatic environment was considered as the reminder of the protein included as fixed MM point charges for rhodopsins and the FMO complex. In the case of the LH2 complex a charge sphere around the $\mathrm{Mg}$ atom with a size of $20 \AA$ was chosen as MM environment. In order to obtain proper thermal averages, structures were extracted from classical (LH complexes) as well as the QM/MM MD trajectories (rhodopsins). Along these 1 ns-long trajectories, 1000 snapshots were used for the computation of the vertical excitation energies.

In the case of rhodopsins, LC-DFTB and OM2/MRCI calculations were employed for the computation of the vertical excitation energies. The OM2/MRCI calculations were carried out using the MNDO2005 program package ${ }^{82,83}$ with an active space of $(20,20) .{ }^{84}$ Due to missing $\mathrm{Mg}$ parameters, the OM2/MRCI approach can presently not be used for the computation of the vertical excitation energies of $\mathrm{LH}$ complexes. In this case, the computation were performed using LC-DFTB and ZINDO/S as detailed above.

\subsection{Exciton couplings}

In a next step we focus on the determination of excitonic couplings. Supermolecular calculations were performed on the model system, Section 3.2.3, using DFT/MRCI with the same setup as described above, HF/CIS with the basis set def2-SVP, TD-DFT (B3LYP) and LC-DFT ( $\omega$ B97X, CAM-B3LYP) with the basis set def2-TZVP employing the TDA as implemented in the ORCA program package. ${ }^{54}$ Furthermore, the RI has been taken into account to speed up the TD-DFT calculations. As in the case of individual chromophores, we used the RIJCOSX for the Coulomb integral and HF exchange and def $2 / \mathrm{J}$ as an auxiliary basis set in the RI approximation. Additionally, supermolecular calculations were performed using TD-DFTB and LC-DFTB.

For LC-DFTB, two different parameter sets have been employed for the computation of Coulomb couplings and supermolecular calculations. The original LC-DFTB parameters reported in ref. 46, yield very good vertical excitation energies and also quite accurate Coulomb couplings, but achieve this by using quite compact atomic orbital basis sets. They were generated by a homogeneous scaling of the original GGA parameter set mio-0-1 of all radii. ${ }^{46}$ It has been shown, however, that using such a confined basis set leads to an underestimation of electron-transfer couplings. ${ }^{85}$ For this type of property, more diffuse basis functions are required since it depends on the overlap of molecular fragments in vdW distance. Therefore, a parameter set was generated using fully uncompressed radii. This parameter set was used for the supermolecular calculations.

For larger inter-chromophore distances, the excitonic couplings can be approximated by the respective Coulomb coupling only. For the model systems, Section 3.2.3, these Coulomb couplings were computed using LC-DFTB based on Mulliken transition charges and an approximation to second order Coulomb interaction described by a $\gamma$-function. ${ }^{45,46}$ We further tested the TrESP approaches (transition charges from electrostatic potentials) ${ }^{41,86}$ as well as a very similar approach based on Mulliken rather than electrostatic charges (Tr-Mulliken). To be able to perform a 
systematic benchmark, we refrained from rescaling the TrESP or Mulliken charges to match the experimental values of the dipole moment as done in the original TrESP scheme. For a discussion of these rescaling and screening factors, see, e.g., ref. 87. The computations of TrESP and Tr-Mulliken charges on the transition densities between ground and first excited state are performed using B3LYP and CAM-B3LYP functionals as implemented in the ORCA program package. Subsequently, the atomic transition charges were obtained using a fitting procedure implemented in the Multiwfn program package. ${ }^{88}$ Additionally, both the TrESP and the Tr-Mulliken charges have been fitted for the actual conformation of one of the two identical monomers. For the TrESP approach this procedure is slightly unusual since the charges are normally fitted for the equilibrium geometry which would potentially lead to some (model) differences between the TrESP and the supermolecular results. Details concerning the computation of the respective Coulomb couplings can be found in the ESI, $\dagger$ see Section S1.2.

In addition, supermolecular calculations were performed directly on a biological system, Section 3.2.4, i.e., on the B850 ring system belonging to the $\mathrm{LH} 2$ complex, where we considered only pairs of neighboring chromophores. The B850 ring consists of so-called $\alpha$ and $\beta$ chromophores which have slightly varying properties due to differences in their environments. Thus the BChla dimer exciton couplings $\mathrm{V}$ are subdivided into two groups denoted as, $c f$. ref. 36: inter-dimer couplings $\mathrm{V}^{1}$, where BChla $\alpha$ and $\beta$ are arranged together with one BChla of the B800 ring, and intra-dimer couplings $\mathrm{V}^{2}$, with only one $\alpha$ - and $\beta$ BChl a chromophore. For the biological system supermolecular computations are performed with LC-DFT and LC-DFTB with the computational setups as described for the model system. For TrESP, however, the charges have been calculated only for the equilibrium conformations and then mapped onto all other conformations. This procedure is sometimes termed "frozen" TrESP approach. ${ }^{89}$ This scheme was chosen here since it is usually employed when computing Coulomb couplings along trajectories, as described above.

\section{Benchmark}

The aim of this section is to investigate the accuracy of LC-DFTB for different model systems. In particular, it is studied whether LC-DFTB can yield qualitatively correct excitation energies for different geometries and point charges used to model a protein environment. In Section 3.1 these influences are investigated for a retinal model, followed by an analysis using BChl a in Section 3.2 for which also excitonic couplings are compared.

\subsection{Retinal}

3.1.1 Bond length alternation. The excitation energies of the chromophore retinal are sensitive to its configuration and correlate with the BLA. An increased BLA leads to a blue shift of the excitation energies. ${ }^{6,18}$ The BLA varies significantly as the geometry is optimized with different QM methods. While BLYP has the smallest BLA, it increases in hybrid approaches with the amount of exact exchange added. HF and CASSCF lead to the highest BLA, see Table S1 in the ESI. $\dagger$ The use of different methods for geometry optimization allows to assess the impact of the BLA on the excitation energies in a systematic manner. This finding is shown in Fig. 3 for all-trans retinal.

SORCI as the reference method displays an expected blue shift that increases with the BLA. ${ }^{18}$ HF-based single-reference methods show the same trend. While CIS overestimates the excitation energies by a factor of about 1.5, SOS-ADC(2) agrees more quantitatively with SORCI, which also applies to OM2/ MRCI. Without spin-component scaling ADC(2) agrees very well with SORCI for small BLA, however, yields a red shift for CASSCF and HF optimized structures $(-0.16 \mathrm{eV})$. GGA, hybrid DFT functionals and TD-DFTB excitation energies are redshifted by up to $0.4 \mathrm{eV}$ with CASSCF geometries.

The $a b$ initio LC functionals, LC-BLYP and $\omega$ B97X, which use $100 \% \mathrm{HF}$ exchange in the long-range limit, obtain the same trend as SORCI, while CAM-B3LYP with only about $60 \% \mathrm{HF}$ exchange still has a qualitative problem. The same trend as described by CAM-B3LYP is obtained by LC-DFTB, however, showing a significant improvement for the HF and CASSCF optimized retinal structures compared to the DFT-GGA and TD-DFTB based on GGA functional. As expected, absolute excitation energies are overestimated by LC functionals.

An important property is the change in excitation energy due to variation in BLA, which shows up, e.g., in the width of the absorption band due to fluctuations. SORCI has a range of $\Delta=0.21 \mathrm{eV}$. SOS-ADC(2) overestimates this range, while ZINDO/S as well as CAM-B3LYP and LC-DFTB underestimate it. A similar range compared to SORCI is obtained by LC-BLYP as well as $\omega \mathrm{B} 97 \mathrm{X}$.

3.1.2 Twist of the $\boldsymbol{\beta}$-ionone ring. Another important geometrical parameter of retinal is the dihedral twist angle of the $\beta$-ionone ring, see Fig. 1. A planar retinal conformation describes a $\pi$-system that is delocalized over the whole conjugated chain, whereas a highly twisted $\mathrm{C}_{6}-\mathrm{C}_{7}$ decouples the ring-internal double bond from the rest of the $\pi$-system, which causes a blue shift, according to the particle-in-a-box model.

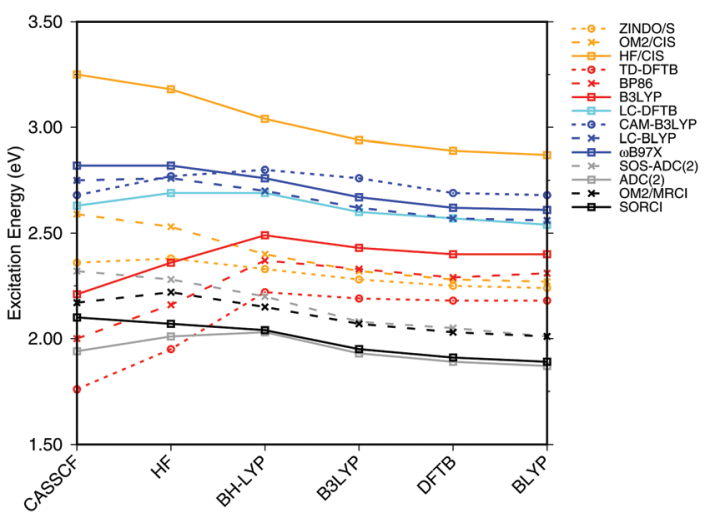

Fig. 3 Influence of the optimization method on the excitation energies: the results for the BP86, DFTB, B3LYP, OM2/CIS, HF/CIS, OM2/MRCl, and $\mathrm{SORCl}$ calculations have been taken from ref. 18. 


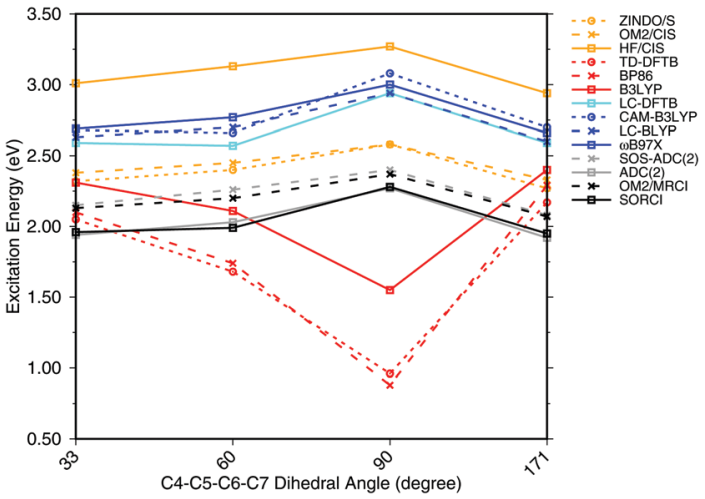

Fig. 4 Excitation energies of a 6-s-cis-11-cis PSB in vacuum for different twist angles. The results for the BP86, DFTB, B3LYP, OM2/CIS, HF/CIS, $\mathrm{OM} 2 / \mathrm{MRCl}$, and SORCl calculations have been taken from ref. 18.

To investigate the effect of delocalization on the excitation energies, a 6-s-cis-11-cis PSB model with different dihedral twist angles has been taken from ref. 18. The results of the methods tested are shown in Fig. 4. The corresponding numerical values can be found in the ESI $\dagger$ (Table S2). ADC(2) shows a very good agreement with SORCI, as well as $\operatorname{SOS}-\operatorname{ADC}(2)$, which is only slightly blue-shifted. Moreover, CIS-based methods follow also the same trend as SORCI with higher absolute excitation energies as obtained in the case of the optimized all-trans retinal structures in Fig. 3. The failure of standard DFT functionals is visible in a red shift of the excitation energies by the retinal twist. As for the BLA, LC-DFT functionals as well as LC-DFTB show an improvement obtaining a blue shift for the twisted retinal configuration.

This failure of hybrid and GGA TD-DFT approaches is even more dramatic as in the case of BLA. In many retinal proteins, i.e., in bovine rhodopsins a blue shift of absorption energy is found due to a twist in the retinal conformation around the $\mathrm{C}_{6}-\mathrm{C}_{7}$ single bond. ${ }^{90}$ The latter is induced by steric interaction of the environment with the retinal chromophore and represents one effective way of color tuning. It is therefore encouraging to see that the LC-DFT methods and LC-DFTB are able to reproduce this feature.

3.1.3 Effect of the electrostatic environment. Retinal proteins absorb light over a wide spectral range of nearly $300 \mathrm{~nm}$, and a major contribution results from electrostatic interactions with the protein environment. The most prominent contribution to the electrostatic interactions with the environment comes from negatively charged groups, i.e., counterions in the binding pocket of the retinal chromophore causing a significant hypsochromic shift of the $S_{1}$ excitation energy. ${ }^{16}$ Depending on the location of the counterions, e.g., near the protonated SB or near the $\beta$-ionone ring, the energy gap varies. For example, the location of the counterion near the protonated SB energetically stabilizes the electronic ground state and thus leads to a larger energy gap (blue shift). ${ }^{91}$

For meaningful benchmarks, a simple counterion model has been proposed, which consists of a PSB5 analog and a single point charge, see Fig. 1 interacting with a point charge in its immediate environment. The PSB5 geometry was reoptimized
Table 1 Vertical excitation energy (eV) of the bare PSB5 model (VEE), shifts due to the presence of a point charge $(-1.1)$

\begin{tabular}{lcccc}
\hline & \multicolumn{4}{c}{ Shifts } \\
\cline { 3 - 5 } Charge $^{b}$ & VEE & $0.0^{c}$ & $-0.5^{c}$ & -1.1 \\
\hline ZINDO/S $^{c}$ & 2.78 & +0.02 & +0.13 & +0.31 \\
TD-DFTB $^{a}$ & 2.77 & -0.09 & +0.00 & +0.11 \\
LC-DFTB & 3.24 & +0.03 & +0.15 & +0.28 \\
CAM-B3LYP & 3.34 & -0.01 & +0.10 & +0.27 \\
LC-BLYP & 3.30 & +0.03 & +0.18 & +0.37 \\
$\omega B 97 X$ & 3.36 & +0.04 & +0.18 & +0.37 \\
SOS-ADC(2) & 2.74 & +0.09 & +0.35 & +0.71 \\
ADC(2) & 2.61 & +0.04 & +0.28 & +0.60 \\
OM2/MRCI $^{a}$ & 2.61 & +0.07 & +0.39 & +0.73 \\
SORCI $^{a}$ & 2.67 & +0.03 & +0.28 & +0.58
\end{tabular}

${ }^{a}$ Taken from ref. $18 .{ }^{b}$ Charges in atomic units. ${ }^{c}$ Vertical excitation energy with downscaled charge but geometry optimized in presence of the full charge of $-1.1 e$.

with DFTB using a charge of -1.1 a.u. at a distance of $2.22 \AA$ to the $\mathrm{SB}$ proton, ${ }^{18}$ which results in a shift when computing the excitation energy of the reoptimized model compared to the model in vacuum. When adiabatically calculating the shift in excitation energy caused by an external field, two effects contribute, (i) the change in geometry and (ii) the interaction of the excitation-induced charge transfer with the external field. When using HF-based methods, these two contributions add up constructively whereas with GGA functionals they can partially cancel out. To show this, we first only include the point charge in the ground-state geometry optimization (column with shift "0.0" in Table 1), then also in the excited-state calculation (columns with shifts "-0.5" and "-1.1”).

SORCI, considered to be the reference method, shows a blue shift of $+0.58 \mathrm{eV}$ with a charge of -1.1 a.u. $\operatorname{ADC}(2)$ and SOS-ADC(2) nicely reproduce this shift, whereas TD-DFT using GGA and hybrid functionals grossly underestimate the shift with values of less than $0.2 \mathrm{eV}$, see ref. 18. TD-DFTB reproduces this failure, and ZINDO/S shifts which are only half of the ones of the SORCI approach. An improvement is observed with LC-DFT functionals as well as LC-DFTB. LC-BLYP and $\omega$ B97X with $+0.4 \mathrm{eV}$ show a larger shift than CAM-B3LYP and LC-DFTB $(+0.3 \mathrm{eV})$. However, the shifts are still substantially smaller, which implies a weakness of the LC functionals. In short, this finding can be termed "color-weakness". In conclusion, TD-DFT with GGA and hybrid functionals are not able to predict color-tuning effects. Furthermore, LC-DFT cannot be considered a quantitative method in this respect neither.

\subsection{Bacteriochlorophyll a (BChl a)}

3.2.1 Geometric and conformational impact on the absorption maximum. Similarly to the first benchmark system, we first investigate the influence of the geometry on excitation energies, in particular on the BLA, see Fig. 5. Different methods for ground state optimization lead to different values of BLA. The computed BLA values are provided in the ESI $\dagger$ (Table S3). In the case of BChla, the use of a multi-reference method like SORCI is impossible due to the large active space required for this chromophore. Thus, we consider DFT/MRCI as a reference, as previously 


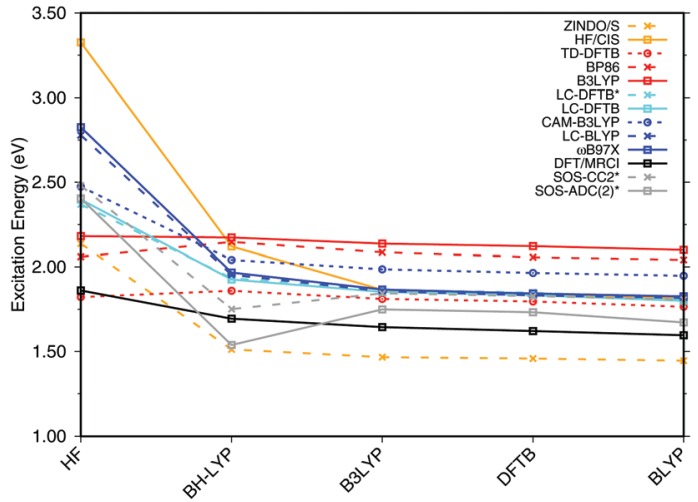

Fig. 5 BChla gas phase excitation energies vs. BLA. The geometries differing in BLA were obtained by using different methods to optimize the ground state structure. The structures are ordered by descending BLA ranging from large (0.102 for HF) to small (0.004 for BLYP). The asterisk * indicates that a truncated structure was used.

suggested in a benchmark study on BChl a, $c f$. ref. 21 . The performance of DFT/MRCI, however, is dependent on the particular Hamiltonian used and the choice of method specific parameters. To consolidate the reference calculations, we therefore also consider the wavefunction-based methods SOS-CC2 and SOS-ADC(2). These are single-reference methods and their reliability is therefore sensitive to the multi-reference character of the ground-state wavefunction, indicated by the $D_{1}$ diagnostic. ${ }^{92}$ However, this $D_{1}$ value is not a strict measure. To avoid distorted structures which lead to a multi-reference ground state, we have, following ref. 26, truncated some substituents, see Fig. 1, which break the symmetry of the BChl a chromophore. When comparing the excitation energies of the truncated and the full geometries using LC-DFTB, we find that the truncation does not lead to significant changes, see $\mathrm{ESI} \dagger$ (Table S3).

Most methods show a continuous blue shift of excitation energies with increasing BLA, with the exception of the GGA and hybrid DFT methods and the ADC and CC variants. For small to intermediate BLA values, all methods perform similarly. This behavior may be relevant when sampling excitation energies along MD simulations, since the BLA changes along the $\mathrm{C}-\mathrm{C}$ stretch modes, which is one of the relevant modes in the molecular dynamics. The blue shift is largest for CIS and ZINDO/S, and seems to depend on the HF-amount in the LC functionals, which becomes apparent when comparing e.g., $\omega \mathrm{B} 97 \mathrm{X}$ and CAM-B3LYP. LC-DFTB shows a behavior quite close to that of CAM-B3LYP, which resembles the change of excitation energy with BLA closely to DFT-MRCI. DFT in general has be considered to been robust with respect to the multi-configurational character, in particular for BChl chromophores, ${ }^{21,27,93}$ it can be concluded that LC-DFT and LC-DFTB are sufficiently accurate for these systems.

3.2.2 Effect of an electrostatic environment. To assess the accuracy of excitation energies with respect to the influence of the protein electrostatic field, QM/MM optimized structures of the FMO complex consisting of seven chromophores, as reported in ref. 21 have been used. For consistency, we recalculated the excitation energies for some methods already reported in ref. 21
(B3LYP, CAM-B3LYP, ZINDO/S) due to the different computational setup. The results show only small changes in the absolute excitation energies while the trends and conclusions from ref. 21 are completely reproduced.

Fig. 6(a) shows the excitation energies of the isolated BChl a chromophores (solid lines) in comparison with the excitation energies calculated including the protein electrostatic field represented by point charges (dashed lines). The corresponding numerical values can be found in the ESI† (Table S4). The axial ligand or the amino acids hydrogen bonded to the BChla chromophore seem to have a small impact on the excitation energy, as reported before..$^{20,21,93}$ This behavior can partially be explained by the transition dipole moment of $6 \mathrm{D}$ of BChl a, ${ }^{94,95}$ which is only about $50 \%$ of the transition dipole moment of retinal (12 D). ${ }^{96}$ B3LYP, CAM-B3LYP as well as LC-DFTB and TD-DFTB lead to accurate vacuum excitation energies, being in agreement with the DFT/MRCI method, which predicts only a small variation in the excitation energies of less than $0.1 \mathrm{eV}$. Slightly larger variations are obtained for LC-DFT methods with larger fractions of HF exchange, e.g., in the case of $\omega \mathrm{B} 97 \mathrm{X}$ for the BChla chromophores 5 and 6 . A significantly different behavior is obtained using $\operatorname{SOS}-\operatorname{ADC}(2)$, being similar to CC2, cf. ref. 21 .

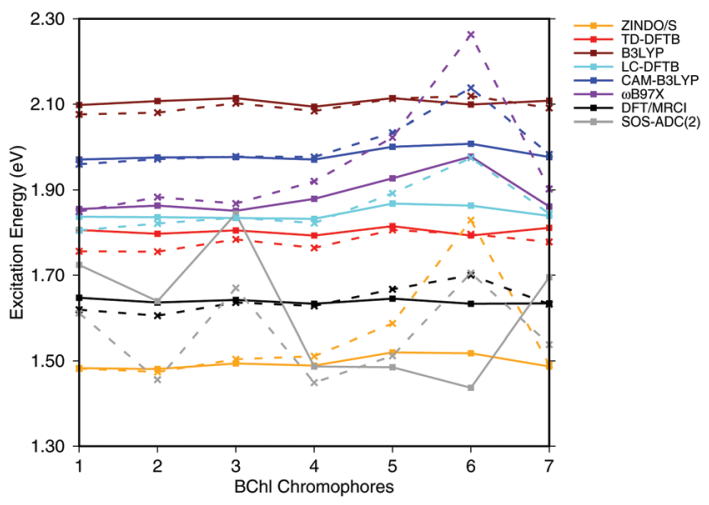

(a)

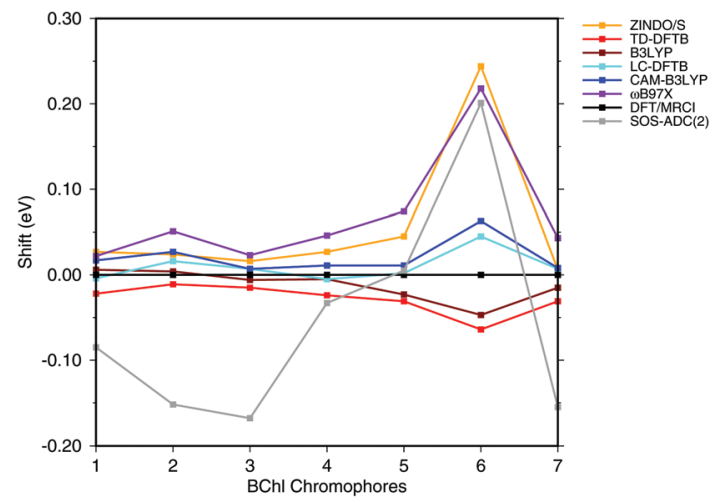

(b)

Fig. 6 (a) Excitation energies of the chromophores from the FMO complex including (solid lines) and not including (vacuum, dashed lines) the surrounding point charges. (b) Shifts of excitation energies due to the protein electrostatic field, displayed with respect to the respective DFT/ $\mathrm{MRCl}$ values. Structures taken from ref. 21. 
To better visualize the behavior, we show the shifts in excitation energies due to the protein environment w.r.t. the DFT/MRCI reference method in Fig. 6(b), values see ESI, $\uparrow$ Table S5. Due to the interaction with the environment, chromophore 6 is the most blue shifted one, and this shift is clearly dependent on the method used. ZINDO/S shows the strongest response to the electrostatic field, as previously reported, $c f$. ref. 21. B3LYP slightly underestimates the shift with respect to DFT/MRCI. For the LC functionals, the amount of exact exchange plays a significant role. For example, $\omega$ B97X leads to a larger shift than CAM-B3LYP, and LC-DFTB reproduces the CAM-B3LYP behavior quite well, deviating only up to $0.05 \mathrm{eV}$ from DFT/MRCI.

3.2.3 Excitonic coupling I: model dimers. In LH complexes the shifting and broadening of the absorption spectra is significantly influenced by the interaction of the excited states of the different BChl a chromophores, also denoted as excitonic coupling. Due to the large separation of the BChl a monomers, both, supermolecular or Coulomb couplings can be used to compute the exciton splittings. In the supermolecular approach, the exchange contribution to the coupling is formally included but in practice is negligible for the systems studied here. ${ }^{97}$

To benchmark LC-DFT as well as LC-DFTB, we first consider a model system consisting of parallel displaced dimers of truncated BChl a chromophores, as described in Section 2.1, to avoid any intra-molecular influences on the exciton coupling. The ADC(2) method is excluded from the assessment of the excitonic couplings due to the large deviations of excitation energies in the protein electrostatic environment compared to all other methods, see Section 3.2.2.

In Table 2 results are collected, which have been obtained from supermolecular calculations for which excitonic coupling are determined as half of the energy splitting of the lowest two states, at least for a homogeneous dimer. The exciton couplings become smaller with increasing distance between the dimers. Compared to DFT/MRCI, CIS slightly overestimates the couplings, while all other DFT methods (hybrid and LC functionals) reproduce the reference quite well. TD-DFTB significantly underestimates the coupling, most likely due to the GGA functional applied, since the absence of exact exchange leads to an underestimation of the energy splittings, a further problem is the state mixing. ${ }^{98}$ LC-DFTB is in good agreement with CAM-B3LYP and improves significantly over TD-DFTB.

Having assessed the accuracy of the supermolecular energy splittings, the same model system is used to investigate the

Table 2 Excitonic couplings (eV) of the model system obtained as half of the energy splitting from supermolecular calculations

\begin{tabular}{lllll}
\hline Distance $(\AA)$ & 7 & 8 & 9 & 10 \\
\hline CIS & 0.069 & 0.051 & 0.040 & 0.031 \\
DFT/MRCI & 0.046 & 0.035 & 0.028 & 0.022 \\
WB97X & 0.059 & 0.044 & 0.034 & 0.027 \\
CAM-B3LYP & 0.058 & 0.044 & 0.034 & 0.026 \\
B3LYP & 0.051 & 0.039 & 0.030 & 0.023 \\
& & & & \\
LC-DFTB & 0.063 & 0.048 & 0.037 & 0.030 \\
TD-DFTB & 0.031 & 0.024 & 0.019 & 0.015
\end{tabular}

Table 3 Coulomb couplings (eV) of the model system using the TrESP, Tr-Mulliken and LC-DFTB schemes. The partial charges of the TrESP and Tr-Mulliken values are based on either the B3LYP and or the CAM-B3LYP functionals

\begin{tabular}{llllll}
\hline & Distance $(\AA)$ & 7 & 8 & 9 & 10 \\
\hline TrESP & B3LYP & 0.050 & 0.038 & 0.029 & 0.023 \\
& CAM-B3LYP & 0.056 & 0.043 & 0.033 & 0.026 \\
\multirow{2}{*}{ Tr-Mulliken } & B3LYP & 0.047 & 0.036 & 0.028 & 0.022 \\
& CAM-B3LYP & 0.054 & 0.041 & 0.032 & 0.025 \\
- & LC-DFTB & 0.082 & 0.063 & 0.049 & 0.039 \\
\hline
\end{tabular}

accuracy of LC-DFTB, TrESP and Tr-Mulliken for Coulomb couplings describing the energy splittings. The Coulomb couplings are collected in Table 3. The TrESP method without any scaling factor can reproduce the corresponding supermolecular results since for the large inter-chromophore distances the exchange contributions can be neglected. This was already reported in a previous study employing the B3LYP functional. ${ }^{87}$ For CAM-B3LYP and B3LYP using TrESP, the Coulomb couplings are, as expected, slightly smaller than those computed with the supermolecular approach. The difference is not big, indicating that the point charge representation of transition densities is a good approximation for these distances. Further, there is no significant difference between ESP and Mulliken charges, indicating that the transition dipole is a quite robust quantity. However, in the case of LC-DFTB it is vice versa. LC-DFTB also uses transition charges computed from a Mulliken charge analysis. In TrESP, these charges are fitted to transition densities using larger basis sets, which seems to be a good approximation. The LC-DFTB Coulomb couplings, however, differ from the Tr-Mulliken calculations in two aspects: first, a minimal basis set is used to compute the Mulliken charges and second, the TD-DFT amplitudes enter the calculation of the Coulomb couplings ${ }^{46}$ as well. LC-DFTB Coulomb couplings possibly can be improved by investigating the applied charge model in more detail.

3.2.4 Exciton coupling II: light harvesting complex II (LH2) B850 ring. The LH2 complex serves as a case study to investigate the role of excitonic interactions in the tuning of an absorption spectrum in real systems. ${ }^{14,99}$ We start with supermolecular calculations on the $16 \mathrm{BChl}$ a dimers (B850 ring), for which results are shown in Fig. 7, the values are collected in the ESI $\dagger$ (Table S6). The results for the biological system show significant differences compared to the model system in Section 3.2.3. Depending on the relative orientation of the $\mathrm{BChl}$ a monomers, the values of the couplings range from about $0.04 \mathrm{eV}$ to about $0.08 \mathrm{eV}$. These values are mostly larger than those in the model system. LC-DFTB values compare quite well with $\omega$ B97X and CAM-B3LYP results. LC-DFTB slightly overestimates the couplings in the model system, which is not the case for the dimers in the B850 ring, and LC-DFTB also reproduces the correct trend of the couplings in the ring system.

Fig. 7 also includes Coulomb couplings. TrESP and Tr-Mulliken methods reproduce the coupling fluctuations along the ring quite well, however, now systematically underestimating the 


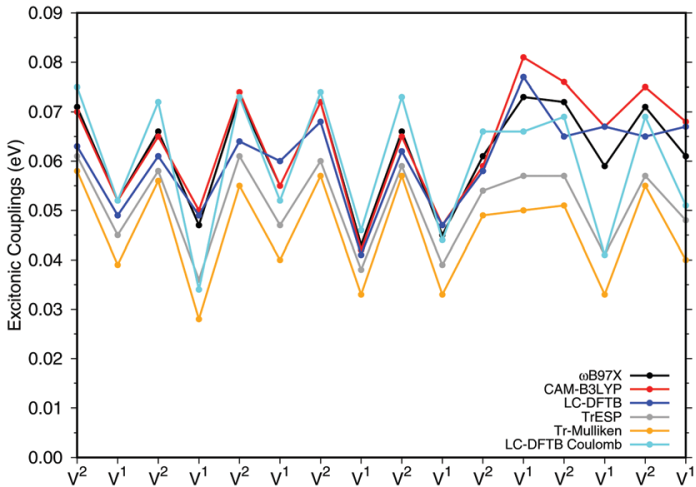

Fig. 7 Excitonic couplings $V(e V)$ for the respective $B C h l a$ dimers $V^{1}$ and $\mathrm{V}^{2}$ of the LH2 B850 ring. The excitonic couplings have been obtained by the supermolecular approach determined as half of the energy splitting The partial charges of the TrESP and Tr-Mulliken schemes are based on CAM-B3LYP

coupling strengths. This may be related to the 'frozen' TrESP approach, where transition charges are only computed for the equilibrium geometry and then mapped to the actual conformer. There is, however, a second factor which may be responsible for the deviation: since exchange effects depend on the mutual overlap of electron densities, which may be dominated by the densities of the highest occupied orbitals, we expect a sensitive geometry dependence of this property. This is less pronounced for the Coulomb couplings, because those are only dependent on the relative orientations of the transition dipole moments. A more detailed study on dimers of organic molecules ${ }^{100}$ seem to support this argument.

Both approaches, TrESP and LC-DFTB, have certain shortcomings, namely the use of transition charges determined for an equilibrium conformation ('frozen' TrESP approach) and the application of a minimal basis set as discussed in Section 3.2.3. However, the mean absolute error (MAE) (see ESI, $\dagger$ Table S6) computed for all methods with respect to the supermolecular $\omega B$ 97X and CAM-B3LYP calculations are small, while LC-DFTB supermolecular as well as LC-DFTB Coulomb couplings show only half of the MAE value compared to TrESP and Tr-Mulliken.
The LC-DFTB Coulomb couplings are in agreement with those obtained from a transition density cube (TDC) approach without an additional scaling factor in ref. 49. A detailed comparison with couplings computed in previous studies, as summarized in Tables S7 and S8 in the ESI, $\dagger$ is difficult due to the frequent use of screening factors.

\section{Performance of LC-DFTB in biological model systems}

In this section, the performance of LC-DFTB on biological model systems, i.e., on rhodopsins and LH complexes is discussed. Due to the efficiency of the LC-DFTB method, not only single-point excitation energies can be computed for minimized geometries but also a statistical average of several thousands structures as obtained by classical or QM/MM MD simulations.

\subsection{Rhodopsin models}

4.1.1 QM/MM optimized models of bR and ppR. In Table 4, we compare the excitation energies of retinal in different electrostatic environments. For each protein, we used the QM/MM optimized structures of bR and ppR from ref. 5, 18 and 101 and show the calculated excitation energies in vacuum (omitting the MM point charges) and within the protein environment, as obtained by different QM methods. All methods agree that without point charges, the excitation energies in $\mathrm{bR}$ and $\mathrm{ppR}$ are very similar and differ by only $0.03 \mathrm{eV}$ on average. This is due to the similar geometry of retinal in the two proteins. When including the protein environment in the calculations, ppR gives a larger blue shift than bR. All methods reproduce this trend, but the magnitude of the shifts varies and reflects the accuracy of the methods in describing the electrostatic influence of the environment. When comparing with the experimental shifts of 0.18 and $0.50 \mathrm{eV}$ for bR and ppR, respectively, all wavefunction based methods overestimate the shift w.r.t. vacuum, whereas DFT based methods underestimate the difference in shift between the two proteins.

The latter observation is consistent with the underestimated response of the model chromophore to external charges,

Table 4 Lowest excitation energies and shifts of QM/MM optimized structures of $\mathrm{bR}$ and $\mathrm{ppR}$, in eV. $\Delta$ denotes the difference of the two systems including the protein environment. The excitation energies are computed only with static external charges without polarization effects leading to higher excitation energies

\begin{tabular}{|c|c|c|c|c|c|c|c|c|c|c|c|}
\hline & Exp. & \multicolumn{6}{|c|}{ Wavefunction } & \multicolumn{2}{|l|}{ DFT } & \multicolumn{2}{|l|}{ DFTB } \\
\hline \multicolumn{12}{|l|}{$\mathrm{bR}$} \\
\hline Protein & $2.18^{b}$ & $2.34^{d}$ & $2.66^{d}$ & 2.48 & 2.76 & 2.35 & 2.64 & 2.94 & 2.85 & 2.77 & 2.22 \\
\hline Shift & +0.18 & +0.48 & +0.44 & +0.40 & +0.51 & +0.46 & +0.51 & +0.29 & +0.21 & +0.19 & +0.18 \\
\hline \multicolumn{12}{|l|}{$\mathrm{ppR}$} \\
\hline Shift & +0.50 & +0.72 & +0.72 & +0.72 & +0.81 & +0.78 & +0.81 & +0.51 & +0.39 & +0.35 & +0.37 \\
\hline$\Delta(\mathrm{ppR}-\mathrm{bR})$ & +0.32 & +0.29 & +0.30 & +0.32 & +0.35 & +0.35 & +0.37 & +0.24 & +0.21 & +0.18 & +0.16 \\
\hline
\end{tabular}

${ }^{a}$ Ref. $104 .{ }^{b}$ Ref. $4 .{ }^{c}$ Ref. $3{ }^{d}$ Ref. 5. 
as described in Section 3.1.3, whereas the first one requires a different explanation. Based on our earlier results, the opsin shift should be underestimated by DFT methods rather than overestimated by all other methods. Here, we refer to previous works, which have shown that point charges obtained by electronic embedding over-polarize the chromophore region. This can be improved by a polarizable embedding. When replacing the point charges in the binding pocket by the electron density of a separate QM region, ${ }^{102}$ or by a polarizable force field, ${ }^{103}$ significantly lower excitation energies are obtained. When the chromophore is described with SORCI and the rest of the protein with the polarization model, excitation energies of 2.16 and $2.42 \mathrm{eV}$ have been reported for bR and $\mathrm{ppR}$, respectively. ${ }^{102,103}$ Compared to vacuum $(2.00 \mathrm{eV}),{ }^{104}$ this corresponds to shifts of 0.16 and $0.42 \mathrm{eV}$, which are in good agreement with the experiment.

The comparison of calculated and measured properties for the purpose of validating theoretical methods is often hampered by experimental measurements in solution. In the present case, the MM modeling of the protein environment introduces errors that can be of the same order of magnitude as those associated with the applied QM methods. Rather than validating, the following discussion therefore attempts to rationalize the results of the different methods and we will employ SORCI shifts as a reference point.

The shifts obtained with OM2/MRCI, CC2, and ADC(2) are in the range of the SORCI ones, with an average deviation of 0.02$0.04 \mathrm{eV}$. The SOS variants of CC2 and ADC(2) yield shifts larger by $0.06 \mathrm{eV}$ on average. Moreover, the LC functionals $\omega \mathrm{B} 97 \mathrm{X}$ and CAM-B3LYP underestimate the shifts by 0.20 and $0.30 \mathrm{eV}$ on average, respectively. TD-DFTB underestimates the shift by even up to $0.35 \mathrm{eV}$ with respect to SORCI. Surprisingly, LC-DFTB does not improve the shifts.

Otherwise, these trends are similar to the ones shown in the previous section for the model chromophore in presence of a single point charge and consistent with previous works (see ref. 18 and references therein), which show that also CIS (based on HF or an OM2 Hamiltonian) underestimates the shift in bR. Therefore, it is not surprising that the shift obtained with LC functionals is larger than that of pure or fixed-hybrid DFT functionals but still significantly lower than that of higher-level methods, like SORCI or CASPT2. Regarding the shifts, the $\omega$ B97X functional performs better than CAM-B3LYP due to its larger amount of HF-X in the long-range limit, but this improvement is paid with a larger error in absolute excitation energies.

4.1.2 QM/MM MD simulation of $b R$ and ppR. A static structure of retinal with its surrounded protein environment obtained by QM/MM optimization misses the fluctuation of the protein environment and the retinal conformations. A QM/MM MD simulation is carried out to sample several structures to assess other influences such as temperature, which are subsequently used for the computation of excitation energies. Hence, the excitation energies are computed on 1000 snapshots using LC-DFTB and OM2/MRCI for bR and ppR, respectively. All excitation energies are weighted by the oscillator strength and plotted as histograms, see Fig. 8(a). The absorption maxima are obtained with a fit using a Gaussian function and displayed in Table 5.

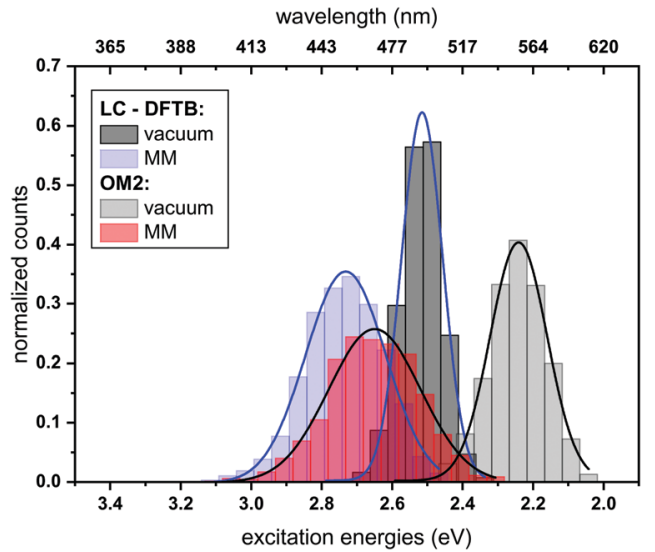

(a)

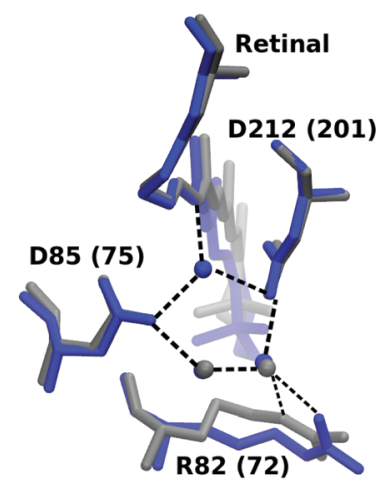

(b)

Fig. 8 (a) Simulated absorption spectrum of bR. LC-DFTB and OM2/MRCI are used for the computations of the excitation energies. The histograms are based on snapshot geometries of QM/MM MD trajectories of 1 ns length. Plotted are the excitation energies weighted by the oscillator strength for (i) only the retinal chromophore (vacuum) and (ii) with additional fixed MM point charges to account for the protein environment (MM). Gaussian functions are used to determine the corresponding maxima, in blue: LC-DFTB and in black: OM2/MRCl. (b) Active site of bR (in blue) and ppR (in gray) with the typical pentagonal hydrogen bond network. Residue numbers of $p p R$ in brackets.

Table 5 Absorption maxima (eV) of $\mathrm{bR}$ and $\mathrm{ppR}$, obtained by sampled excitation energies over the QM/MM MD trajectories with subsequent Gaussian fit of the histograms. (ppR - bR) denotes the difference of the two systems in the protein environment

\begin{tabular}{llcc}
\hline & Exp. & OM2/MRCI & LC-DFTB \\
\hline bR & & & \\
Vacuum & - & 2.24 & 2.52 \\
Protein & $2.18^{a}$ & 2.65 & 2.73 \\
Shift & - & +0.41 & +0.21 \\
& & & \\
ppR & - & 2.26 & 2.53 \\
Vacuum & $2.50^{b}$ & 2.98 & 2.93 \\
Protein & - & +0.72 & +0.40 \\
Shift & +0.32 & +0.33 & +0.20 \\
& & & \\
A(ppR - bR) & & & \\
${ }^{a}$ Ref. $4 .{ }^{b}$ Ref. 3. & & & \\
\hline
\end{tabular}

The absorption maxima of the retinal chromophore in bR and $\mathrm{ppR}$ in vacuum differ by about $\pm 0.02 \mathrm{eV}$ for each method. 
This result is expected, since the retinal conformations are similar in bR and ppR. In comparison with the QM/MM optimized models, OM2/MRCI displays a slight blue shift of $+0.02 \mathrm{eV}$ and LC-DFTB a red shift of about $-0.06 \mathrm{eV}$ for the vacuum excitation energies. The same behavior is obtained for the absorption maxima including the protein environment.

In $\mathrm{bR}$ and $\mathrm{ppR}$ the inclusion of the protein environment results in a blue shift for both methods. In the case of bR, OM2/ MRCI leads to a slightly smaller blue shift $(0.41 \mathrm{eV})$ compared to the QM/MM optimized models $(0.44 \mathrm{eV})$. Whereas in the case of ppR, LC-DFTB computes a slightly higher blue shift $(0.40 \mathrm{eV})$ compared to the QM/MM optimized models $(0.35 \mathrm{eV})$. The slight deviations compared to the QM/MM optimized models of both rhodopsins bR and ppR is mainly reflected by the strong hydrogen bond network (see Fig. 8(b)) in the active site confining the chromophore retinal in its structural fluctuation. In this case the QM/MM MD simulations describe only an oscillation of the chromophore retinal around the energy minimum. ${ }^{5}$ However, it has to be mentioned that rhodopsins exist, e.g., channelrhodopsin-2 wild-type (ChR2-WT), exhibiting a very flexible structure and therefore needs sampling by QM/MM MD simulations. ${ }^{84}$ The difference of the absorption maxima between both rhodopsins (ppR-bR) obtained by OM2/MRCI $(+0.33 \mathrm{eV})$ is as expected in agreement to the experimental shift $(+0.32 \mathrm{eV})$. LC-DFTB shows with $+0.20 \mathrm{eV}$ a smaller shift of the absorption maxima between $\mathrm{bR}$ and ppR. Here, the color-weakness of LC-DFTB is again visible.

The computed excitation energies of the sampled structures are plotted as histograms in Fig. 8(a) for bR. The histogram for ppR is shown in the ESI $\dagger$ (Fig. S3). In the case of OM2/MRCI, a broad simulated absorption spectrum is obtained, while LC-DFTB tends to yield less broad absorption spectra, especially when considering only the retinal chromophore without the protein environment. The width of the absorption spectra reflects in the case of vacuum, the different obtained retinal conformations differing in their BLA. The inclusion of the protein environment includes then also the fluctuation of the protein itself. Hence, weaknesses of LC-DFTB become visible also in this property since it cannot reproduce the same width as OM2/MRCI.

\subsection{Light-harvesting complexes}

To asses the performance of LC-DFTB, we focus on the energy ranges of three LH complexes, LH2 complex of Rs. molischianum and Rps. acidophila and the FMO complex of C. tepidum. To this end, we analyze the energy range spanned by all individual uncoupled BChl a chromophores with and without the protein environment as well as the energy range of the excitons.

\subsubsection{Light-harvesting complex II (LH2)}

QM/MM optimized model. In a first step, we considered a DFTB-QM/MM optimized structure for the complex and computed excitation energies for the B800 and B850 ring systems using LC-DFTB as summarized in Table 6. More detailed information can be found in the ESI $\dagger$ (Tables S9 and S10). The BChl a structures are not significantly influenced by the protein environment and thus the excitation energies show only a minute standard deviation of $0.01 \mathrm{eV}$ due to structural effects. The electrostatic interaction based on the QM/MM point charge model leads to a larger
Table 6 Average excitation energies and standard deviations (in eV) based on DFTB-QM/MM optimized BChl a structures of the B800 and B850 ring systems. Shown are the excitation energies for the individual BChla chromophores with and without the influence of the protein environment. In addition, excitonic energies (in eV), i.e., the lowest eigenvalues are shown for the ring system. The excitation energies have been computed using LC-DFTB, Coulomb couplings with LC-DFTB and TrESP

\begin{tabular}{llll}
\hline & B800 & B850 & B850 shift \\
\hline Vacuum $^{a}$ & $1.829 \pm 0.007$ & $1.829 \pm 0.004$ & \pm 0.000 \\
Protein $^{a}$ & $1.837 \pm 0.028$ & $1.828 \pm 0.011$ & -0.009 \\
& & & \\
Ring system & & 1.740 & -0.055 \\
LC-DFTB & 1.795 & 1.730 & -0.064 \\
TrESP & 1.794 & & -0.085 \\
Experimental & & & \\
${ }^{a}$ BChl a monomer. $^{109}$ & & \\
\hline
\end{tabular}

variation of the excitation energies, which is also illustrated in Fig. 9. It leads to a larger range for the B800 ring $(0.092 \mathrm{eV})$ than for the $\mathrm{B} 850 \mathrm{ring}(0.034 \mathrm{eV})$. This finding can be explained by a more pronounced influence of the protein environment in B800, since the chromophores are less densely packed than in B850 and thereby exposed to a more polar protein environment. ${ }^{105,106}$ As expected from the benchmark results (Section 3), ZINDO/S overestimates the effect of the electrostatic tuning and results in a larger variation as shown in Table S11, ESI. $\dagger$

LC-DFTB Coulomb couplings are about $0.006 \mathrm{eV}$ for neighboring BChla in a B800 ring, while we find a range of 0.033$0.075 \mathrm{eV}$ for the B850 ring. TrESP gives smaller Coulomb couplings, as expected from the benchmark study (Section 3), which are around $0.004 \mathrm{eV}$ for the B800 ring and $0.038-0.061 \mathrm{eV}$ for the B850 ring. The excitonic energies resulting from the diagonalization of the Hamiltonian for the two individual ring systems show a range of excitonic energies of $0.096 \mathrm{eV}$ for $\mathrm{B} 800$, and $0.274 \mathrm{eV}$ for B850. A similar energy range of $0.317 \mathrm{eV}$ for the B850 ring was already reported previously. ${ }^{107}$ Although for LH2 of Rs. molischianum no experimental data is known regarding the excitonic energy range, experimental data for the bacterium Rps. acidophila suggests an energy range of about $0.179 \mathrm{eV}$ for the 8850 ring at low temperatures. ${ }^{108}$ Therefore, LC-DFTB overestimates this range of energies, and the reason for this overestimation is two-fold: first, the spread of the excitation energies of the individual chromophores may be slightly overestimated due to the QM/MM point charge electrostatics, i.e., inclusion of polarizable embedding may damp this spread slightly. ${ }^{36,102,103}$ Second, LC-DFTB overestimates the values of the Coulomb couplings, as seen in Section 3. The Coulomb couplings are larger than the LC-DFTB supermolecular couplings, which in turn are in very good agreement with full LC-DFT couplings. Moreover, effects of polarization on the couplings are neglected. But all these effects are small $(0.01-0.02 \mathrm{eV})$, but they accumulate when determining the eigenvalues of the Hamiltonian of the ring system. Therefore, we expect that our approach overestimates the spread of eigenvalues by about $0.1 \mathrm{eV}$.

In order to estimate the B800-B850 energy splitting, we use the lowest eigenvalues of the two ring Hamiltonians. As shown 


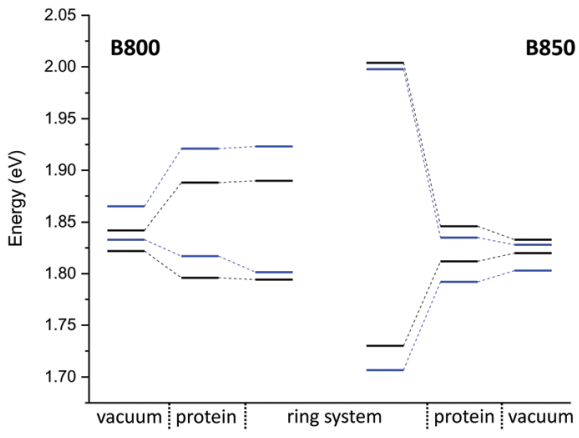

Fig. 9 Excitation energy range (vacuum and with electrostatic environment) combined with the excitonic energy range for the QM/MM optimized structures, computed using LC-DFTB. LH2 of Rs. molischianum in black and LH2 of Rps. acidophila in blue.

in Table 6, this range is close to the experimental value, i.e., the errors cancel out to some extent. The reason can be understood by inspecting Fig. 9. On the one hand, the Coulomb couplings are overestimated, partially due to neglect of polarization. This significantly affects the B850 excitonic energies, i.e., additionally lowering the lowest energy states. Since the couplings between the $\mathrm{B} 800$ chromophores are much smaller, this spectrum is not much affected. The neglect of polarization, on the other hand, also lowers the energies of the individual chromophores, i.e., the site energies of the excitonic Hamiltonian: this, as discussed above, affects mostly B800 energies. Therefore, the energies of both ring systems are artificially lowered due to computational errors, i.e., for the B800 the site energies are mostly affected, for B850 the couplings. Since in both cases, the excitonic energies are lowered, we find a cancellation of errors.

The computation of such small energy differences, i.e., below $0.1 \mathrm{eV}$, is very challenging for these large systems, where approximations are inevitable. A recent study ${ }^{36}$ on the LH2 complex of Rps. acidophila reported an overestimation of the experimental gap, while the computed excitonic couplings are smaller than ours (around $0.05 \mathrm{eV}$ ) due to the consideration of polarization effects. Since it is interesting to see how different approaches perform for the same system, we also studied the LH2 complex of Rps. acidophila. A detailed discussion and comparison of methods can be found in the ESI. $\dagger$ The monomer excitation and excitonic energies of this complex are shown in Fig. 9. Although the two LH2 complexes studied here differ in conformation and number of chromophores, the spectra are quite similar.

Classical MD simulation. QM/MM MD simulations for multichromophoric complexes are not straightforward, since a QM approach with multiple QM regions would be required which is not implemented in the present DFTB scheme. Therefore, we use classical MD trajectories to sample the excitation energies. The corresponding average excitation energies and standard deviations are shown in Table 7. Moreover, in Fig. 10 histograms based on snapshots of the $\mathrm{BChl}$ a chromophores belonging to the $\mathrm{B} 800$ ring using a $1 \mathrm{~ns}-\mathrm{long} \mathrm{MD}$ trajectory are displayed. The results for the $\mathrm{B} 850$ ring are given in the $\mathrm{ESI}^{\dagger}$ (Fig. S4). While the vacuum excitation energy distribution is similar for LC-DFTB
Table 7 Average excitation energies and standard deviations (in eV) based on the classical MD trajectories. Shown are the excitation energies for the individual BChla chromophores with and without the influence of the protein environment. The average excitation energies are obtained by averaging the maxima of Gaussian fits of the distributions of the respective chromophore. In addition, the excitonic energies (in eV), i.e., the lowest eigenvalues are shown for the Hamiltonians considering Coulomb couplings between the BChla chromophores within one ring system. The excitonic energies are extracted as the maxima of the Gaussian fits to the exciton energy distributions. The excitation energies have been computed using LC-DFTB, Coulomb couplings with LC-DFTB and TrESP

\begin{tabular}{llll}
\hline & B800 & B850 & B850 shift \\
\hline Vacuum $^{a}$ & $1.833 \pm 0.003$ & $1.835 \pm 0.005$ & -0.002 \\
Protein $^{a}$ & $1.844 \pm 0.007$ & $1.839 \pm 0.002$ & -0.005 \\
Ring system & & & \\
LC-DFTB & 1.815 & 1.737 & -0.078 \\
TrESP & 1.817 & 1.771 & -0.046 \\
Experimental $^{109}$ & & -0.085 \\
${ }^{a}$ BChla monomer. & & \\
\hline
\end{tabular}

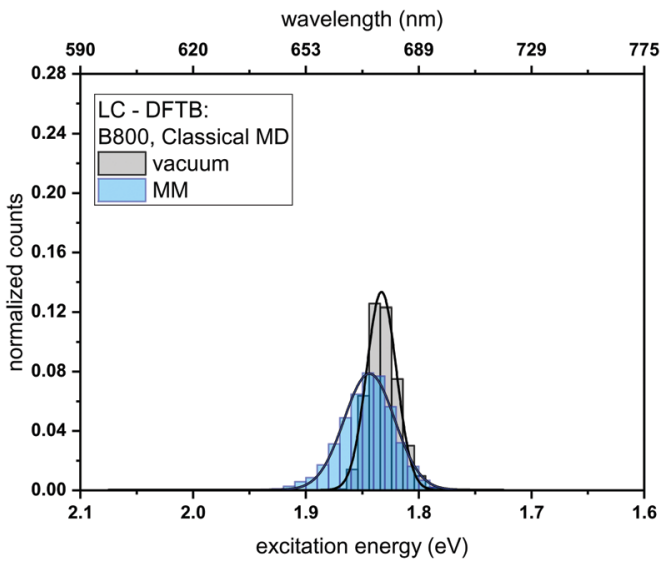

(a)

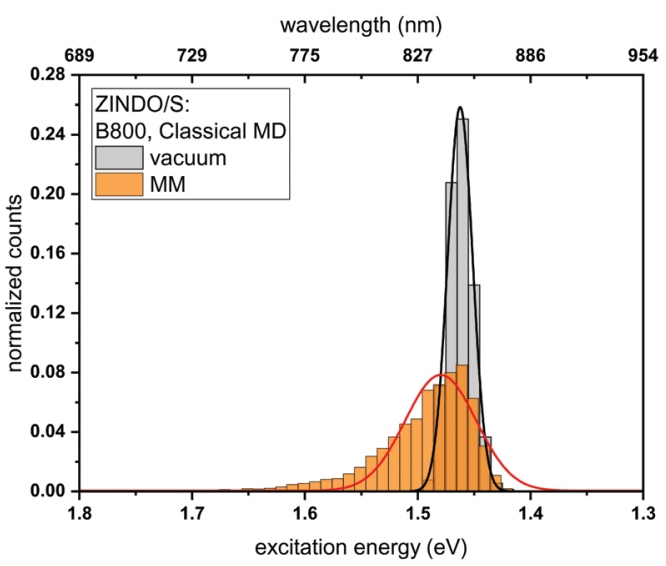

(b)

Fig. 10 Histogram of the excitation energies for the $\mathrm{B} 800$ ring computed with (a) LC-DFTB and (b) ZINDO/S. Plotted are the excitation energies weighted by the oscillator strength for (i) only the BChla chromophore (vacuum) and (ii) with additional fixed MM point charges to account for the protein environment (MM). Gaussian functions have been used to determine the corresponding maxima. 


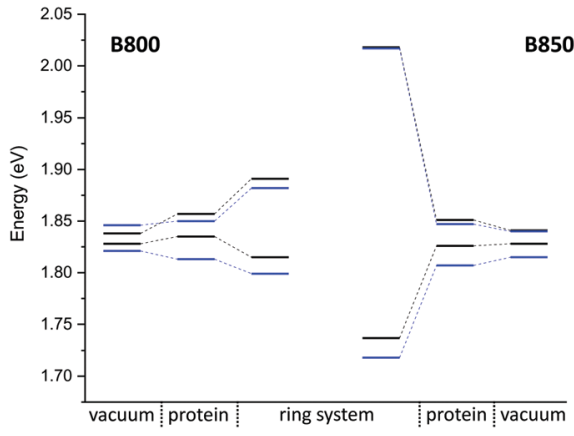

Fig. 11 Excitation energy range of maxima of Gaussian fits (vacuum and with electrostatic environment) combined with excitonic energy range (in eV) for the sampled structures, computed with LC-DFTB. LH2 of Rs. molischianum in black and LH2 of Rps. acidophila in blue.

and ZINDO/S, the different response of both methods to the electrostatic field of the protein, as discussed in Section 3, is clearly visible. Both methods show a broadening while $\mathrm{ZINDO} / \mathrm{S}$ shows a strongly asymmetric distribution with a long tail towards higher energies as reported earlier. ${ }^{41,110}$

Compared to the QM/MM optimized structures, the standard deviations (Table 7) are reduced for the average excitation energies with and without the protein environment. For LC-DFTB, the diagonal disorder, i.e., the range of excitation energies, is smaller compared to that of the QM/MM optimized structures, as can be seen from Fig. 11. It seems, that optimization leads to local minima, where excitation energy differences between the BChl a chromophores are more pronounced. Hence, the differences between both LH2 complexes are reduced, comparing Fig. 11 and 9 (more details, see Table S15, ESI $\dagger$ ).

The sampled Coulomb couplings over the classical MD trajectory clearly show differences when comparing LC-DFTB and TrESP. An example plot is given in the ESI $\dagger$ (Fig. S5). The LC-DFTB couplings fluctuate in a range of $0.004-0.008 \mathrm{eV}$ for B800 and $0.060-0.080 \mathrm{eV}$ for B850, while the TrESP couplings show lower fluctuations of $0.0018-0.0025 \mathrm{eV}$ for B800 and $0.035-$ $0.045 \mathrm{eV}$ for $\mathrm{B} 850$. The variation arises only from geometric changes, since the LC-DFTB computed couplings undergo changes due to changes in the transition charges, while in the case of TrESP the transition charges are constant. Please note, that we found the TrESP approach to lead to smaller couplings already for the model systems presented above. The B800-B850 energy splitting estimated from the sampled structures is higher in the case of LC-DFTB compared to the QM/MM optimized model and closer to the experimental value. As discussed above, error cancellation might also be responsible for the better agreement with the experimental energy range.

The resulting excitonic energy range computed using LC-DFTB decreases after sampling compared to the QM/MM optimized model in the case of B800, while it slightly increase in the case of B850 (see ESI, $\dagger$ Table S14). As discussed for the QM/MM optimized model, the diagonal disorder is dominant in the B800 ring, since the couplings do not contribute much. Since the excitation energy splitting of the protein environment decreased after sampling, the excitonic splitting decreased as well. In the case of B850, smaller differences are observed compared to the QM/MM optimized model. For B850, the off-diagonal disorder is smaller through the more tight packing ${ }^{105,106}$ (see also discussion above), the chromophores are less exposed to the fluctuations of the protein electrostatic field. Thus, at the LC-DFTB level, there is no significant change in the exciton splitting when compared to the QM/MM optimized case, which can be seen by comparing Fig. 11 and 9. For the TrESP couplings, however, a sizable decrease is found upon sampling, where the exciton splitting of $0.163 \mathrm{eV}$ is closer to the experimental value at room temperature of about $0.158 \mathrm{eV} .{ }^{108}$ This however, may be an accidential agreement, since the TrESP couplings are much smaller than those from supermolecular calculations.

One has to note, however, that the averaging process for the excitonic energies is ambiguous in itself: one can average the Hamiltonian and then compute its eigenvalues or one can average the excitonic energies computed for every snapshot. The latter one is used in the present work. Since the diagonalization is a non-linear operation, the two approaches lead to different results (Table S14, ESI $\dagger$ ). Thus, these two averaging procedures do not commute. In the first case, the deviations from the average value will not receive an appropriate weight in the exciton formation, while in the second case an instantaneous response of the electronic structure to the nuclear motion is assumed. This is a time-scale problem occurring typically, when a transport process is coupled to nuclear motions: how to treat fluctuations, e.g., in the context of Marcus theory ${ }^{111}$ or Landauer tunneling. ${ }^{112}$ When the time scales of the fluctuations in the electronic structure, resulting for the nuclear motion, and transport of the charge or energy are on the same time scale, non-adiabatic methods seem to be more appropriate.

\subsubsection{Fenna-Matthews-Olson (FMO) complex}

$Q M / M M$ optimized model. Next we analyze the excitation energies of the individual $\mathrm{BChl}$ a chromophores from the FMO of C. tepidum. In Fig. 12, the results for the DFTB QM/MM optimized FMO complex are depicted, computed in vacuum and within the protein environment using LC-DFTB. As observed for the LH2 complex, the BChla geometries are not significantly influenced by the protein environment, since the BLA values are in the same range. Furthermore, the ZINDO/S results show a stronger response to the electrostatics (see ESI, $\dagger$ Table S18).

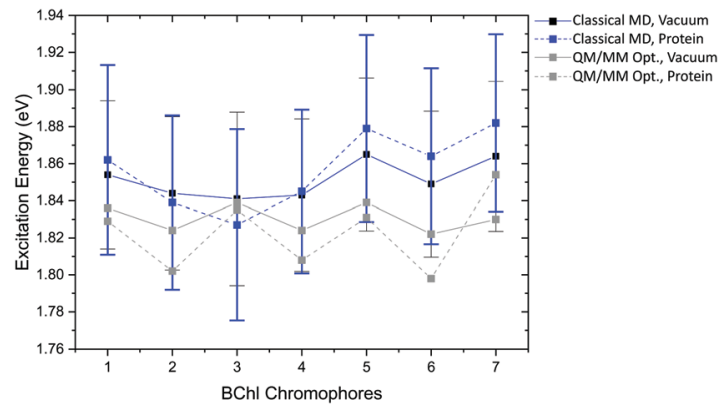

Fig. 12 Excitation energy range of the FMO complex of the seven BChla chromophores of the DFTB QM/MM optimized model and maxima with error bars of the Gaussian distributions belonging to the sampled structure over the classical trajectory; calculated in vacuum and with fixed MM point charges using LC-DFTB. 
Table 8 Range of excitation and excitonic energies of the DFTB QM/MM optimized BChl a chromophores of the FMO complex. LC-DFTB is used for the computation of excitation energies without (vacuum) and with the protein environment as fixed MM point charges. Coulomb couplings are computed using LC-DFTB and TrESP

\begin{tabular}{llll}
\hline & Max & Min & Shift \\
\hline Vacuum $^{a}$ & 1.839 & 1.822 & -0.017 \\
Protein $^{a}$ & 1.854 & 1.798 & -0.056 \\
& & & \\
Coupled chromophores & & & \\
LC-DFTB & 1.898 & 1.756 & -0.142 \\
TrESP & 1.968 & 1.784 & -0.184 \\
& & & -0.060 \\
Experimental & & & \\
${ }^{a}$ BChl a monomer. & 1.563 & 1.503 & \\
\end{tabular}

The respective minimum and maximum of the energy range obtained in vacuum and with the protein environment are presented in Table 8. As expected, the resulting energy range in vacuum of $0.017 \mathrm{eV}$ is smaller than the experimental range of $0.060 \mathrm{eV} \cdot{ }^{113}$ The consideration of the electrostatic environment increases this value to $0.056 \mathrm{eV}$. A previous study using QM/MM optimized structures of the FMO complex of $P$. aesturii reporting an energy range of about $0.025 \mathrm{eV}$ using CAM-B3LYP and including polarization contribution. ${ }^{48}$ Therefore, using point charges only, the protein electrostatic effect may be overestimated, since polarization is neglected here.

For LC-DFTB Coulomb couplings, a range of values between $0.019 \mathrm{eV}$ and $0.044 \mathrm{eV}$ is obtained for the strongest couplings, i.e., for the neighboring BChl a chromophores. In the case of TrESP a range of $0.012-0.029 \mathrm{eV}$ is found showing lower values than LC-DFTB as expected from the benchmark study, see Section 3. The Hamiltonian and the excitonic energies of the QM/MM optimized model computed with LC-DFTB are given in the ESI $\dagger$ (Tables S17 and S21, ESI $\dagger$ ). The resulting energy range between the maximum and the minimum of the excitonic energies overestimates the experimental energy range, see Table 8, as expected, due to the overestimation of the Coulomb couplings and the neglect of polarization effects, similar to the LH2 case discussed above.

Classical MD simulation. In a next step, we analyze the excitation energies for the single BChl a chromophores sampled along a classical MD trajectory. The maxima of Gaussian fits to the distributions with the respective standard deviations are plotted in Fig. 12. LC-DFTB was used for the computation of the excitation energies of the sampled structures with and without protein environment. Again, ZINDO/S shows a stronger response to the electrostatic environment as shown by the broader excitation energy distributions depicted in the ESI $\dagger$ (Fig. S9-S11). However, the standard deviations of both LC-DFTB and ZINDO/S are similar, showing values of about $0.048 \mathrm{eV}$. The vacuum excitation energy range of the BChl a chromophores is in the case of LC-DFTB slightly larger $(0.024 \mathrm{eV})$ than for the QM/MM optimized model $(0.017 \mathrm{eV})$, see Table 9 due to the fluctuations.

The energy range computed for the protein environment is similar to that obtained by the QM/MM optimized model (see
Table 9 Range of excitation and excitonic energies along a classical MD trajectory of the FMO complex. LC-DFTB is used for the computation of excitation energies without (vacuum) and with the protein environment. The Coulomb couplings have been computed using LC-DFTB and TrESP

\begin{tabular}{llll}
\hline & Max & Min & Shift \\
\hline Vacuum $^{a}$ & 1.865 & 1.841 & -0.024 \\
Protein $^{a}$ & 1.882 & 1.827 & -0.055 \\
Coupled chromophores & & & \\
LC-DFTB & 1.979 & 1.775 & -0.204 \\
TrESP & 1.876 & 1.777 & -0.099 \\
Experimental $^{113}$ & 1.563 & 1.503 & -0.060 \\
${ }^{a}$ BChl a monomer. & & & \\
\end{tabular}

Tables 8 and 9). The results are consistent with those from a previous study $c f$. ref. 114, which also did not include polarization effects. As already mentioned polarization effects reduce this range to $0.025 \mathrm{eV}^{115}$

The average Coulomb couplings sampled along the trajectories are slightly smaller than for the QM/MM optimized model, see ESI $\dagger$ (Table S19). These are plotted together with Coulomb couplings obtained by previous studies using TD-DFT/MMPol ${ }^{115}$ and PDA, ${ }^{116}$ see $\mathrm{ESI}^{\dagger} \dagger$ (Fig. S8). LC-DFTB obtains qualitatively the same trend, however overestimates the Coulomb couplings by about a factor of 2. In the case of FMO, the Coulomb couplings seems to be slightly more overestimated than for the LH2 complex, when comparing to previous studies. ${ }^{36,49}$ Protein polarization leads to a screening of electrostatic interactions, which can be represented by a screening factor specific for the chromophores in different protein environments. Jurinovich et al., ${ }^{115}$ reported a screening factor of about 1.7 for the FMO complex, which is larger than the screening factor obtained for the LH2 complex of $1.47 .{ }^{36}$ This indicates a higher influence of polarization, which explains the different deviations of our results.

The energy range resulting from the average excitonic energies (see ESI, $\dagger$ Table S21), i.e., the exciton energy range from the coupled FMO chromophores shown in Table 9, is larger than the experimental energy range. It is even larger compared to the energy range obtained by the QM/MM optimized model. In the case of FMO, the off-diagonal disorder is dominant, leading to an increase of the exciton energy range compared to the QM/MM optimized model. Furthermore, the Coulomb couplings might have been overestimated more severely in the FMO complex compared to the LH2 aggregate due to the above discussed polarization influence.

\section{Summary}

In this study, we have investigated the performance of LC-DFT and LC-DFTB for two major light-activated protein classes, rhodopsins and light-harvesting complexes. Both present significant challenges for a theoretical description: rhodopsins show a large variance in absorption energies, i.e., have a sophisticated mechanism of color tuning depending on chromophore geometry and interaction with the environment, while light-harvesting complexes 
are less responsive to the interaction with the environment, but are large in size and to a large extent change color by interchromophore coupling. For both systems, standard DFT functionals give non-satisfactory results while range-separated functionals seem to be a promising alternative.

For retinal, GGA and hybrid DFT functionals both fail to describe color tuning w.r.t. changes in geometry and protein electrostatic interactions, while LC-DFT and LC-DFTB, in contrast, give a quite good account for geometrical changes. However, the correct response to the protein electrostatic field is sensitively dependent on the amount of exact exchange. Therefore, the $\omega \mathrm{B} 97 \mathrm{X}$ and LC-BLYP functionals come close to the reference methods and might be useful in applications, while for CAM-B3LYP and LC-DFTB the response to the protein electrostatic field is too weak, therefore, these methods are not suitable for studies of retinal protein color tuning.

For BChla, the changes of absorption energies w.r.t. geometries are well reproduced by LC-DFT and LC-DFTB. Electrostatic effects are much smaller than in retinal proteins but still relevant, since small energy differences lead to sizable effects in the red part of the spectrum. Taking DFT/MRCI as reference, it seems that less exact exchange in the LC-DFT functionals leads to a better agreement, so that CAM-B3LYP and LC-DFTB yield comparably the best results. Therefore, CAM-B3LYP might be the LC reference method of choice. For the calculation of excitonic couplings in BChla, the choice of the LC-DFT functional is not critical, and they are also quite well described by LC-DFTB. Coulomb couplings computed from LC-DFTB, however, are slightly overestimated.

Therefore, the LC functionals have to be chosen carefully according to the target systems, since the correct description of absorption energies of retinal and $\mathrm{BChl}$ a depends on the amount of exact exchange. This is in inherited by LC-DFTB due to the particular choice of the LC functional.

The low computational cost of semi-empirical methods allows for extensive sampling of absorption spectra along MD trajectories, but also for a direct propagation of the exciton wavefunction on relevant time-scales. ${ }^{46}$ We have performed QM/MM MD simulations to sample rhodopsin absorption energies using LC-DFTB and OM2/MRCI as fast quantum methods. While OM2/MRCI performs quite well, LC-DFTB computed spectra have to be considered carefully, due to the too small response to the protein electrostatic fields.

Considering the LH complexes, in a first step we have QM/MM optimized their structures iteratively, by successively selecting one chromophore in the QM region. The calculated excitation energies for these QM/MM optimized structures indicate, that the diagonal disorder in the excitonic Hamiltonian is overestimated. This leads to an overestimation of the width of the computed spectrum. It seems, that geometry optimization drives the geometries into local minima, which show a large variation of excitation energies among the chromophores. These may be shallow minima, which seem to contribute less when averaging over trajectories at $300 \mathrm{~K}$. A problem, which can be solved by either imposing symmetry ${ }^{36}$ or by averaging. We tested this for both LH complexes studied here, showing a clear reduction of energy range differences after sampling. We sampled the excitation spectra using trajectories from classical MD, since QM/MM MD simulations for the entire LH complexes are not possible at the moment. A multi-chromophore QM region would be required, work is in progress to make this property available in DFTB.

The comparison of exciton splittings for both LH2 and FMO computed with LC-DFTB and ZINDO/S show clear differences: since ZINDO/S shows a stronger response to the electrostatic environment, the width of the exciton spectrum is overestimated. LC-DFTB seems to be a good alternative method to ZINDO/S representing the electrostatic interaction in a more balanced way. Up to now, effects of protein polarization, which we included in our previous studies on retinal proteins ${ }^{101-103}$ have not been taken into account. However, chromophore excitation energies and exciton couplings are affected by polarization. Both, diagonal disorder and the coupling strength are slightly reduced when considering protein polarization effects, therefore, not taking polarization into account leads to an overestimation of the exciton bandwidth.

Interestingly, the experimental B800-B850 energy splitting is reproduced quite well, since polarization effects tend to cancel. This is not the case for the FMO complex, where the width of the exciton band is overestimated. Therefore, LC-DFTB will most likely give quite accurate results for $\mathrm{LH}$ complexes excited states properties, if polarization is included in future work.

In summary, LC-DFTB is an accurate and efficient method for studying exciton spectra and dynamics in $\mathrm{LH}$ complexes, since it is about three orders of magnitude faster than the full TD-DFT scheme with a minimum loss in accuracy.

\section{Conflicts of interest}

The authors declare no competing financial interest.

\section{Acknowledgements}

The authors acknowledge support by the DFG through the joint grant EL 206/18-1 and KL-1299/18-1, the DFG-Research Training groups 2247 “Quantum Mechanical Materials Modelling”, 2039 "Molecular architecture for fluorescent cell imaging”, 2450 "Tailored Scale-Bridging Approaches to Computational Nanoscience" and the DFG-SFB 1249 "N-Heteropolyzyklen als Funktionsmaterialien" (Projects B02 and B07). Moreover, support by the state of BadenWürttenberg through bwHPC and the German Research Foundation (DFG) through grant no INST 40/467-1 FUGG (JUSTUS cluster) is gratefully appreciated. The project has been supported in part by the bwHPC initiative and the bwHPC-C5 project. We thank N. H. List for providing us with the structures and benchmark data of the FMO complex from ref. 21 .

\section{Notes and references}

1 S. L. Logunov, L. Song and M. A. El-Sayed, J. Phys. Chem., 1996, 100, 18586-18591.

2 G. G. Kochendoerfer, S. W. Lin, T. P. Sakmar and R. A. Mathies, Trends Biochem. Sci., 1999, 24, 300-305. 
3 I. Chizhov, G. Schmies, R. Seidel, J. R. Sydor, B. Lüttenberg and M. Engelhard, Biophys. J., 1998, 75, 999-1009.

4 R. R. Birge and C. F. Zhang, J. Chem. Phys., 1990, 92, 7178-7195.

5 M. Hoffmann, M. Wanko, P. Strodel, P. H. Konig, T. Frauenheim, K. Schulten, W. Thiel, E. Tajkhorshid and M. Elstner, J. Am. Chem. Soc., 2006, 128, 10808-10818.

6 M. Wanko, M. Hoffmann, T. Frauenheim and M. Elstner, J. Comput. Aided Mol. Des., 2006, 20, 511-518.

7 R. E. Blankenship, Molecular Mechanisms of Photosynthesis, John Wiley \& Sons, 2014.

8 M. Gouterman, J. Mol. Spectrosc., 1961, 6, 138-163.

9 M. Gouterman and G. H. Wagnière, J. Mol. Spectrosc., 1963, 127, 108-127.

10 M. Umetsu, Z. Wang, K. Yoza, M. Kobayashi and T. Nozawa, Biochim. Biophys. Acta, 2000, 1457, 106-117.

11 E. Thyrhaug, K. Žídek, J. Dostál, D. Bína and D. Zigmantas, J. Phys. Chem. Lett., 2016, 7, 1653-1660.

12 J. Neugebauer, ChemPhysChem, 2009, 10, 3148-3173.

13 M. T. Milder, B. Brüggemann, R. van Grondelle and J. L. Herek, Photosynth. Res., 2010, 104, 257-274.

14 C. Curutchet and B. Mennucci, Chem. Rev., 2017, 117, 294-343.

15 M. Schreiber and V. Buss, Int. J. Quantum Chem., 2003, 95, 882-889.

16 N. Ferré and M. Olivucci, J. Am. Chem. Soc., 2003, 125, 6868-6869.

17 A. J. A. Aquino, M. Barbatti and H. Lischka, ChemPhysChem, 2006, 7, 2089-2096.

18 M. Wanko, M. Hoffmann, P. Strodel, A. Koslowski, W. Thiel, F. Neese, T. Frauenheim and M. Elstner, J. Phys. Chem. B, 2005, 109, 3606-3615.

19 A. Anda, T. Hansen and L. De Vico, J. Chem. Theory Comput., 2016, 12, 1305-1313.

20 A. Anda, L. De Vico and T. Hansen, J. Phys. Chem. B, 2017, 121, 5499-5508.

21 N. H. List, C. Curutchet, S. Knecht, B. Mennucci and J. Kongsted, J. Chem. Theory Comput., 2013, 9, 4928-4938.

22 R. Send and D. Sundholm, Phys. Chem. Chem. Phys., 2007, 9, 2862-2867.

23 R. Send and D. Sundholm, J. Phys. Chem. A, 2007, 111, 27-33.

24 R. Send, D. Sundholm, M. P. Johansson and F. Pawlowski, J. Chem. Theory Comput., 2009, 5, 2401-2414.

25 O. Valsson and C. Filippi, J. Chem. Theory Comput., 2010, 6, 1275-1292.

26 C. M. Suomivuori, N. O. Winter, C. Hättig, D. Sundholm and V. R. Kaila, J. Chem. Theory Comput., 2016, 12, 2644-2651.

27 J. Linnanto and J. Korppi-Tommola, Phys. Chem. Chem. Phys., 2006, 8, 663-687.

28 D. Jacquemin, E. A. Perpète, G. Scalmani, M. J. Frisch, R. Kobayashi and C. Adamo, J. Chem. Phys., 2007, 126, $0-12$.

29 D. Jacquemin, E. A. Perpète, G. E. Scuseria, I. Ciofini and C. Adamo, J. Chem. Theory Comput., 2008, 4, 123-135.

30 I. V. Rostov, R. D. Amos, R. Kobayashi, G. Scalmani and M. J. Frisch, J. Phys. Chem. B, 2010, 114, 5547-5555.
31 Y. Tawada, T. Tsuneda, S. Yanagisawa, T. Yanai and K. Hirao, J. Chem. Phys., 2004, 120, 8425-8433.

32 O. Valsson, C. Filippi and M. E. Casida, J. Chem. Phys., 2015, 142, 144104.

33 T. Yanai, D. P. Tew and N. C. Handy, Chem. Phys. Lett., 2004, 393, 51-57.

34 M. Higashi, T. Kosugi, S. Hayashi and S. Saito, J. Phys. Chem. B, 2014, 118, 10906-10918.

35 T. Wolter, M. Elstner, S. Fischer, J. C. Smith and A.-N. Bondar, J. Phys. Chem. B, 2015, 119, 2229-2240.

36 L. Cupellini, S. Jurinovich, M. Campetella, S. Caprasecca, C. A. Guido, S. M. Kelly, A. T. Gardiner, R. Cogdell and B. Mennucci, J. Phys. Chem. B, 2016, 120, 11348-11359.

37 A. D. Bacon and M. C. Zerner, Theor. Chim. Acta, 1979, 53, 21-54.

38 M. C. Zerner, G. H. Loew, R. F. Kirchner and U. T. MuellerWesterhofflc, J. Am. Chem. Soc., 1980, 102, 589-599.

39 M. G. Cory, M. C. Zerner, X. Hu and K. Schulten, J. Phys. Chem. B, 1998, 102, 7640-7650.

40 C. Olbrich, T. L. C. Jansen, J. Liebers, M. Aghtar, J. Strümpfer, K. Schulten, J. Knoester and U. Kleinekathöfer, J. Phys. Chem. B, 2011, 115, 8609-8621.

41 C. Olbrich and U. Kleinekathöfer, J. Phys. Chem. B, 2010, 114, 12427-12437.

42 K. Welke, J. S. Frähmcke, H. C. Watanabe, P. Hegemann and M. Elstner, J. Phys. Chem. B, 2011, 115, 15119-15128.

43 T. Niehaus, THEOCHEM, 2009, 914, 38-49.

44 T. A. Niehaus and F. Della Sala, Phys. Status Solidi B, 2012, 249, 237-244.

45 J. J. Kranz, M. Elstner, B. Aradi, T. Frauenheim, V. Lutsker, A. D. Garcia and T. A. Niehaus, J. Chem. Theory Comput., 2017, 13, 1737-1747.

46 J. J. Kranz and M. Elstner, J. Chem. Theory Comput., 2016, 12, 4209-4221.

47 L. Stojanović, S. G. Aziz, R. H. Hilal, F. Plasser, T. A. Niehaus and M. Barbatti, J. Chem. Theory Comput., 2017, 13, 5846-5860.

48 C. Steinmann and J. Kongsted, J. Chem. Theory Comput., 2015, 11, 4283-4293.

49 F. Segatta, L. Cupellini, S. Jurinovich, S. Mukamel, M. Dapor, S. Taioli, M. Garavelli and B. Mennucci, J. Am. Chem. Soc., 2017, 139, 7558-7567.

50 R. Ahlrichs, M. Bär, M. Häser, H. Horn and C. Kölmel, Chem. Phys. Lett., 1989, 162, 165-169.

51 A. Schäfer, H. Horn and R. Ahlrichs, J. Chem. Phys., 1992, 97, 2571-2577.

52 A. Schäfer, C. Huber and R. Ahlrichs, J. Chem. Phys., 1994, 100, 5829-5835.

53 M. Elstner, D. Porezag, G. Jungnickel, J. Elsner, M. Haugk, T. Frauenheim, S. Suhai and G. Seifert, Phys. Rev. B: Condens. Matter Mater. Phys., 1998, 58, 7260-7268.

54 F. Neese, Wiley Interdiscip. Rev.: Comput. Mol. Sci., 2018, 8, 4-9.

55 J. Almlöf, T. H. Fischer, P. G. Gassman, A. Ghosh and M. Häser, J. Phys. Chem., 1993, 97, 10964-10970.

56 M. J. Abraham, T. Murtola, R. Schulz, S. Páll, J. C. Smith, B. Hess and E. Lindahl, SoftwareX, 2015, 1-2, 19-25. 
57 T. Kubař, K. Welke and G. Groenhof, J. Comput. Chem., 2015, 36, 1978-1989.

58 M. Eichinger, P. Tavan, J. Hutter and M. Parrinello, J. Chem. Phys., 1999, 110, 10452-10467.

59 H. C. Watanabe, K. Welke, D. J. Sindhikara, P. Hegemann and M. Elstner, J. Mol. Biol., 2013, 425, 1795-1814.

60 J. Huang and A. D. MacKerell, J. Comput. Chem., 2013, 34, 2135-2145.

61 M. Gaus, Q. Cui and M. Elstner, J. Chem. Theory Comput., 2011, 7, 931-948.

62 M. Gaus, A. Goez and M. Elstner, J. Chem. Theory Comput., 2013, 9, 338-354.

63 M. Gaus, X. Lu, M. Elstner and Q. Cui, J. Chem. Theory Comput., 2014, 10, 1518-1537.

64 B. Hess, C. Kutzner, D. van der Spoel and E. Lindahl, J. Chem. Theory Comput., 2008, 4, 435-447.

65 A. D. MacKerell, D. Bashford, M. Bellott, R. L. Dunbrack, J. D. Evanseck, M. J. Field, S. Fischer, J. Gao, H. Guo, S. Ha, D. Joseph-McCarthy, L. Kuchnir, K. Kuczera, F. T. K. Lau, C. Mattos, S. Michnick, T. Ngo, D. T. Nguyen, B. Prodhom, W. E. Reiher, B. Roux, M. Schlenkrich, J. C. Smith, R. Stote, J. Straub, M. Watanabe, J. Wiórkiewicz-Kuczera, D. Yin and M. Karplus, J. Phys. Chem. B, 1998, 102, 3586-3616.

66 C. S. López, O. N. Faza, S. L. Estévez and A. R. De Lera, J. Comput. Chem., 2006, 27, 116-123.

67 S. Hirata and M. Head-Gordon, Chem. Phys. Lett., 1999, 314, 291-299.

68 K. Eichkorn, O. Treutler, H. Öhm, M. Häser and R. Ahlrichs, Chem. Phys. Lett., 1995, 242, 652-660.

69 F. Neese, F. Wennmohs, A. Hansen and U. Becker, Chem. Phys., 2009, 356, 98-109.

70 F. Weigend, Phys. Chem. Chem. Phys., 2006, 8, 1057-1065.

71 J. D. Chai and M. Head-Gordon, J. Chem. Phys., 2008, 128, 084106.

72 T. A. Niehaus, S. Suhai, F. Della Sala, M. Elstner, G. Seifert and T. Frauenheim, Phys. Rev. B: Condens. Matter Mater. Phys., 2001, 63, 1-9.

73 V. Lutsker, B. Aradi and T. A. Niehaus, J. Chem. Phys., 2015, 143, 184107.

74 R. Baer and D. Neuhauser, Phys. Rev. Lett., 2005, 94, $2-5$.

75 E. Livshits and R. Baer, Phys. Chem. Chem. Phys., 2007, 9, 2932-2941.

76 C. M. Marian, A. Heil and M. Kleinschmidt, Wiley Interdiscip. Rev.: Comput. Mol. Sci., 2019, 9, e1394.

77 S. Maity, A. Gelessus, V. Daskalakis and U. Kleinekathöfer, Chem. Phys., 2019, 526, 110439.

78 C. Hättig and F. Weigend, J. Chem. Phys., 2000, 113, 5154-5161.

79 C. Hättig, Adv. Quantum Chem., 2005, 50, 37-60.

80 N. O. C. Winter and C. Hättig, J. Chem. Phys., 2011, 134, 184101.

81 N. O. C. Winter, N. K. Graf, S. Leutwyler and C. Hättig, Phys. Chem. Chem. Phys., 2013, 15, 6623-6630.

82 A. Koslowski, M. E. Beck and W. Thiel, J. Comput. Chem., 2003, 6, 714-726.
83 W. Weber and W. Thiel, Theor. Chem. Acc., 2000, 103, 495-506.

84 Y. Guo, F. E. Beyle, B. M. Bold, H. C. Watanabe, A. Koslowski, W. Thiel, P. Hegemann, M. Marazzi and M. Elstner, Chem. Sci., 2016, 7, 3879-3891.

85 A. Kubas, F. Hoffmann, A. Heck, H. Oberhofer, M. Elstner and J. Blumberger, J. Chem. Phys., 2014, 140, 104105.

86 M. E. Madjet, A. Abdurahman and T. Renger, J. Phys. Chem. B, 2006, 110, 17268-17281.

87 E. P. Kenny and I. Kassal, J. Phys. Chem. B, 2016, 120, 25-32.

88 T. Lu and F. Chen, J. Comput. Chem., 2012, 33, 580-592.

89 H. Kitoh-Nishioka, D. Yokogawa and S. Irle, J. Phys. Chem. C, 2017, 121, 4220-4238.

90 S. Ahuja, M. Eilers, A. Hirshfeld, E. C. Y. Yan, M. Ziliox, T. P. Sakmar, M. Sheves and S. O. Smith, J. Am. Chem. Soc., 2009, 131, 15160-15169.

91 L. H. Andersen, I. B. Nielsen, M. B. Kristensen, M. O. El Ghazaly, S. Haacke, M. B. Nielsen and M. A. Petersen, J. Am. Chem. Soc., 2005, 127, 12347-12350.

92 C. L. Janssen and I. M. Nielsen, Chem. Phys. Lett., 1998, 290, 423-430.

93 J. Neugebauer, J. Phys. Chem. B, 2008, 112, 2207-2217.

94 R. S. Knox and B. Q. Spring, Photochem. Photobiol., 2003, 77, 497-501.

95 R. G. Alden, E. Johnson, V. Nagarajan and W. W. Parson, J. Phys. Chem. B, 1997, 101, 4667-4680.

96 R. Mathies and L. Stryer, Proc. Natl. Acad. Sci. U. S. A., 1976, 73, 2169-2173.

97 P. A. Plötz, T. Niehaus and O. Kühn, J. Chem. Phys., 2014, 140, 174101.

98 T. Kubař, P. B. Woiczikowski, G. Cuniberti and M. Elstner, J. Phys. Chem. B, 2008, 112, 7937-7947.

99 B. Moore and J. Autschbach, ChemistryOpen, 2012, 1, 184-194.

100 N. Schieschke, B. M. Bold, D. Holub, M. Hoffman, A. Dreuw, M. Elstner and S. Höfener, to be published.

101 J. S. Frähmcke, M. Wanko, P. Phatak, M. A. Mroginski and M. Elstner, J. Phys. Chem. B, 2010, 114, 11338-11352.

102 M. Wanko, M. Hoffmann, T. Frauenheim and M. Elstner, J. Phys. Chem. B, 2008, 112, 11462-11467.

103 M. Wanko, M. Hoffmann, J. Frähmcke, T. Frauenheim and M. Elstner, J. Phys. Chem. B, 2008, 112, 11468-11478.

104 I. B. Nielsen, L. Lammich and L. H. Andersen, Phys. Rev. Lett., 2006, 96, 13-16.

105 A. Damjanović, I. Kosztin, U. Kleinekathöfer and K. Schulten, Phys. Rev. E, 2002, 65, 1-24.

106 L. Janosi, I. Kosztin and A. Damjanović, J. Chem. Phys., 2006, 125, 14903.

107 A. Damjanović, T. Ritz and K. Schulten, Phys. Rev. E, 1999, 59, 3293-3311.

108 M. Pajusalu, M. Rätsep, G. Trinkunas and A. Freiberg, ChemPhysChem, 2011, 12, 634-644.

109 J.-p. Zhang, R. Fujii, P. Qian, T. Inaba, T. Mizoguchi, Y. Koyama, K. Onaka, Y. Watanabe and H. Nagae, J. Phys. Chem. B, 2000, 104, 3683-3691. 
110 S. Chandrasekaran, M. Aghtar, S. Valleau, A. Aspuru-Guzik and U. Kleinekathöfer, J. Phys. Chem. B, 2015, 119, 9995-10004.

111 S. S. Skourtis, D. H. Waldeck and D. N. Beratan, Annu. Rev. Phys. Chem., 2010, 61, 461-485.

112 P. B. Woiczikowski, T. Kubař, R. Gutiérrez, R. A. Caetano, G. Cuniberti and M. Elstner, J. Chem. Phys., 2009, 130, 215104.
113 D. Hayes and G. S. Engel, Biophys. J., 2011, 100, 2043-2052. 114 C. W. Kim, B. Choi and Y. M. Rhee, Phys. Chem. Chem. Phys., 2017, 20, 3310-3319.

115 S. Jurinovich, C. Curutchet and B. Mennucci, ChemPhysChem, 2014, 15, 3194-3204.

116 X. Jia, Y. Mei, J. Z. H. Zhang and Y. Mo, Sci. Rep., 2015, 5, $1-10$. 